\title{
A Survey of Smart Hydroponic Systems
}

\author{
Falmata Modu $^{1}$, Adam Adam $^{*} 1$, Farouq Aliyu ${ }^{2}$, Audu Mabu ${ }^{1}$, Mahdi Musa $^{1}$ \\ ${ }^{1}$ Department of Computer Science, Yobe State University, 620242, Nigeria \\ ${ }^{2}$ Department of Computer Engineering, King Fahd University of Petroleum and Minerals, 34464, Saudi Arabia
}

A R T I C L E I N F O

Article history:

Received: 03 December, 2019

Accepted: 04 January, 2020

Online: 29 January, 2020

Keywords:

Hydroponics

Internet of Things

Smart Cities
A B S T R A C T

The explosion in human population has left researchers scrambling for solutions on how to feed the world. Furthermore, rural-urban immigration has on the one hand left the farms in the rural areas devoid of farmers and on the other hand has left the urban areas over-populated. Hydroponics is a form of agriculture where crops are grown without soil. This technique allows the farms to follow the farmers to the urban area. In addition, the fact that no soil is needed, allows hydroponic system to be stacked vertically (also known as vertical farming) to save space. The final frontier in hydroponics is automation. It will allow one farmer to work more than one job and cultivate more than one farm simultaneously. This paper provides a comprehensive survey on smart hydroponic system developed to date.

\section{Introduction}

The soil is a valuable component of agriculture [1]: it provides support for the plants, it also provide nutrient to the plants and the soil provide a home to some of the microbial organism that forms a symbiosis relationship with the plants. However, all these ingredients can be provided with hydroponics. Hydroponics is the process of growing plants without soil [2, 1]. Evidence of hydroponics was found in the Egyptian wall painting [3]. There are many benefits to hydroponics [1]: 1) it does not require soil, 2) it is faster than traditional farming, 3)it requires less space and can be grown in any location, 4) it is unaffected by seasonal change, 5) little or no pesticides and herbicides are needed 6) Plants get complete range of nutrients they need at the quantity they need it, 7) Plants are protected against diseases and pests, 8) It can be used to isolate crops during experiments [4, 5, 6, 7].

Figure 1 shows the components of a hydroponic system: First there is a need for a location or growing area where the system is going to be installed since hydroponics require only water any space could be used for it. The reservoir is a container that stores the nutrient solution used by the system. Nutrients in a good hydroponic system must contain the optimum level of; oxygenation, salinity, $\mathrm{pH}$, and conductivity of nutrient solution [8]. The hydroponics fertilizers contain six essential nutrients: $\mathrm{N}, \mathrm{P}, \mathrm{S}, \mathrm{K}, \mathrm{Ca}$ and $\mathrm{Mg}$, which are fed to the plants in form of mutual ratio of anions: $\mathrm{NO}_{3}^{-}, \mathrm{H}_{2} \mathrm{PO}_{4}^{-}$ and $\mathrm{SO}_{4}^{2-}$, and the mutual ratio of cations $\mathrm{K}^{+}, \mathrm{Ca}^{2+}$ and $\mathrm{Mg}^{2+}$ [9]. Several growing medium like a wood chip can be used with water to form a hydroponics system [10]. Light is necessary for photosynthesis, in close/indoor hydroponic systems light-emitting diodes (LEDs) and other sources of light are used to provide lighting in lieu of the sun. Other factors that may be considered are; ambient temperature, nutrient solution temperature, photoperiod, and air humidity [8].

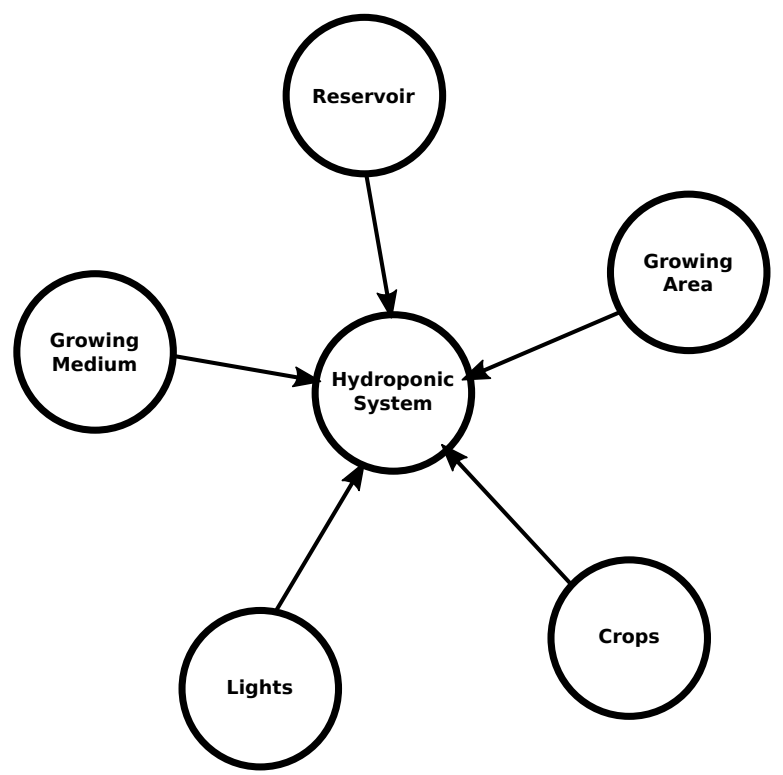

Figure 1: Components of a hydroponic system

${ }^{*}$ Corresponding Author Adam Adam, Department of Computer Science, Yobe State University, (+234) 8060673838 \& adam@ysu.edu.sa 


\subsection{Types of Hydroponics}

Figure 2 shows a basic hydroponic system - all hydroponic systems can be developed by modifying it. Hydroponic techniques are divided into seven types, Wick, Deep Water Culture (DWC), Ebb and Flow (Flood and Drain), Drip (recovery or non-recovery), Nutrient Film Technique (NFT), Aeroponics [11] and Fogponics.

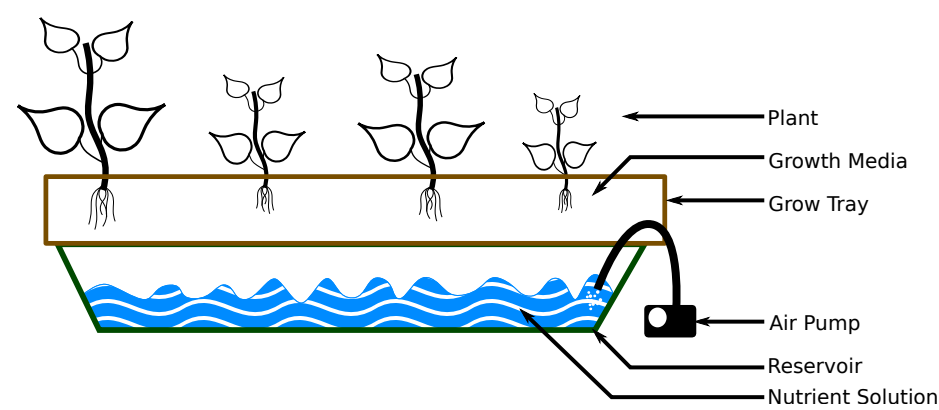

Figure 2: A Hydroponic system

\subsubsection{Wick Hydroponic}

As the name implies, the wick type hydroponics system feeds the plants with the nutrient solution via a wick. The most widely used wicks are Pro-Mix, Vermiculite, Perlite and Coconut Fiber [12]. The system is easy to maintain because it is a passive system without any moving parts not even an air pump since the plants' roots are not submerged in the nutrient solution. However, its biggest weakness is that the wick can only deliver a small quantity of water to the plants at a time. Hence, bigger plants may be starved.

\subsubsection{Deep Water Culture (DWC) Hydroponics}

DWC is also known as water culture hydroponics. In this system, the growth medium is made up of Styrofoam, which floats directly on the nutrient solution. Oxygen delivery is the difficult part of this system [13]. Since the plants' roots are completely submerged in the nutrients a system of air pump and air stone provides them with oxygen. DWC is commonly used in plants the need a large quantity of water to grow, such as lettuce.

\subsubsection{EBB and Flow (Flood and Drain) Hydroponics}

In this system, a submersible pump is installed in the nutrient solution where it pumps the solution up and into the growth tray to flood it. A system is installed such that it allows the solution to ebb back into the reservoir. The pump is controlled with a timer such that it turns on the pump to fill the growth tray and then turn it off so that the solution flows back slowly into the reservoir. This flood-ebb cycle allows the root to get a solution (during the flood cycle) as well as oxygen (during the ebb cycle). However, plants' root are susceptible to diseases [14]. Since the plants' life entirely depend on the flood-ebb cycle, then by extension it means they depend on the timer, the pump and the drain system, which significantly reduces the reliability of the system.

\subsubsection{Drip Hydroponic}

Here the system uses drip to feed the plants with the nutrients from the reservoir. Excess nutrients are either fed-back into the reservoir or it is allowed to drain away or evaporate. The earlier is known as recovery drain hydroponics while the latter is called a non-recovery drain hydroponic system [15]. The advantage of this system is that it can be tailored to any type of plant since the flow rate of the nutrient can be adjusted. However, it is difficult to maintain because of the $\mathrm{pH}$ shift in the recovery type.

\subsubsection{Nutrient Film Technique (NFT)}

NFT pumps nutrient solution to a tilted growth tray. The nutrient flow to the other end of the tray where it is drained back to the reservoir. The system requires no growth medium and timer. Furthermore, the air pump is not needed since the growth medium of the system is air. Unfortunately, the plants are prone to wilting when the flow of nutrient flow stops because the roots dry relatively fast [16].

\subsubsection{Aeroponics}

This system gets rid of the barrier between the growth tray and the reservoir. The roots are left dangling in the reservoir above the solution. A mist of the solution is sprayed to the roots at regular intervals. Aeration is the primary advantage of this system. Also, a smaller amount of nutrient is consumed compared to other systems [17]. However, the system needs a shorter time interval for the nutrient cycle. This is tantamount to more energy consumption. Moreover, the plants' root would quickly dry should the pump or timer fails.

\subsubsection{Fogponics}

A more advanced variant of aeroponics is Fogponics. Here too, the growth medium is air. However, fog emitter (also known as fogger) is used to produce smaller droplets (ranging $5-30 \mu \mathrm{m}$ ) than in aeroponics. The fog produced delivers water and nutrients to the plants' root. Fogponics is better than aeroponics because; smaller (fog) droplets encourage more nutrient absorption [18], the fog can also reach more parts of the plants' root compared to spray droplets and the lack of flowing nutrient solution allows for easier crop monitoring [19].

\subsection{Motivation}

A lot of work has been carried out to improve and/or automate hydroponic systems: Sambo et. al [20], presents a literature survey of hydroponic systems and smart technologies that can be used to improve the performance of such systems. Kaewwiset and Yooyativong [9] investigated the relationship between the electrical conductivity (EC) and the $\mathrm{pH}$ of the hydroponic nutrient mixing system. In [21], potassium acrylate is proposed for hydroponic substrates, because of its water-absorbent nature and the fact that it is biodegradable. In another research [22], the best method to reduce power consumption while maintaining optimum growth is investigated. 
The authors found that Far Red (FR) treatment leads to late flowering and slow stem growth. The impact of microalgae on hydroponics is investigated in [23]. The authors argue that they can be used to purify wastewater which can be used in hydroponics.

In this paper, the latest effort of researchers to automate the hydroponic system using embedded systems and the Internet of Things (IoT) is reported. In addition, a nomenclature of a smart hydroponic system base on the studied literature is presented. The remaining part of the paper is as follows: Section 2 presents an analysis of the smart hydroponic systems in the literature and developed a nomenclature for them. Section 3 lists the challenges in smart hydroponics and recommends some possible research areas. Section 4 concludes the paper.

\section{Smart Hydroponic Systems}

Automated hydroponic systems have been investigated in the literature for a long time. This is because the automated systems have the advantage of providing huge throughput, high efficiency and require no specialized manpower. Furthermore, the automation of hydroponic is not beyond reach since the tasks carried out in a hydroponic system can easily be automated. Also, computers have the capacity to do it better, because of its high availability compare to humans.

In this paper, the smart hydroponic systems are classified into four (4) main categories. The categories are developed based on the Automation level, Tasks automated, Type of Automation and Mode of control:

\subsection{Automation Level}

This section explores the classification of hydroponic systems based on the level of automation. Hydroponics can be classified as; 1) semi-automated, and 2) fully automated. The earlier is a group of hydroponics systems that control only part of the system shown in Figure 1, while the latter is a group that covers all hydroponics system where all its components are automatically controlled.

\subsubsection{Semi-automated}

Savvas [24], proposed two alternative techniques for automatic nutrient replenishment in closed-loop hydroponic systems. Both proposed systems use electric conductivity and $\mathrm{pH}$ as input and addition of nutrients or freshwater as output. The first system uses several pumps (one for each nutrient and another for freshwater) while the second has only two pumps (one for fertilizer and the other for freshwater). The author concluded that both systems can be used in replenishing nutrients in hydroponic systems. Also, Domingues et al. [25], developed an Automated Hydroponic System (AHS) that uses $\mathrm{pH}$, temperature and electric conductivity as parameters for measuring the nutrients level. They argue that temperature is a very important parameter since it is directly related to both $\mathrm{pH}$ and electric conductivity. The system is used in growing lettuce (Lactuca sativa L.). The proposed AHS uses a system of electric valves, one for each nutrient. They are used to control the $\mathrm{pH}$ level of the nutrient solution. The authors show that the proposed system is as good as hydroponics system control by experts.
Phutthisathian et. al [26] developed an Ontology-based nutrient solution control system for hydroponics was developed. The authors argue that building a smart hydroponic system is a complex task and it should be designed using Ontology Web Language (OWL) and Resource Description Framework (RDF). The system controls the nutrient supply through two nutrient tanks and $\mathrm{pH}$ levels through the use of a separate acid tank. The system also has a fourth tank for water supply. The system uses EC to monitor the nutrient solution. The authors build an ontology of control variables from OWL and RDF. Ontology is able to design structure and mechanism that makes hydroponic systems more effective.

\subsubsection{Fully Automated}

Yamaguchi et. al [27] developed a small closed hydroponic system capable of cultivating 10 heads of lettuce is developed in this paper. In this paper, a closed hydroponic system is a hydroponics system designed to be used indoors or in a glasshouse, where lighting could be controlled. The system has sensors for monitoring the temperature and humidity of the system and its outside surroundings. It also has a light sensor for measuring the ambient light. Also, the system has white LEDs, a cooling fan, an air pump to supply oxygen to the nutrient solution. A Raspberry Pi with a camera is also installed to observe the system. The authors also used renewable energy to manage the hydroponic system. A hybrid energy control system using lead-acid battery and hydrogen fuel cell battery was deployed. The proposed system has the potential of bringing hydroponic farming to every home, because of its ease of setup. Furthermore, the use of renewable energy reduces the carbon footprint of the system. The authors were able to harvest the lettuce after 28 days. Also, the coexistence of the system and occupants prove fruitful due to the abundant $\mathrm{CO}_{2}$ provided by the later. The proposed system requires human intervention in order to run efficiently.

Aquaponics is a type of hydroponic system that uses aquaculture (fish farming) as the main source of nutrients from the fish wastes. The plants remove the nitrogen and phosphorous introduced in the water by the fish thus cleaning the water. In this paper [28], an automated closed (i.e. indoor) aquaponics is developed. Managed by an Arduino Mega microcontroller, the system feeds the fish, monitors and controls the water temperature and level. The pump is used to irrigate the Water spinach (Ipomoea aquatica) and Spinach (Spinacia oleracea) used in the system. In addition, the stand-alone system uses an SD card to keep a log of the activities of the microcontroller. However, the system depends on fixed parameters set by the user, also the system uses a 100W light source which may increase the temperature of the aquaponic system.

\subsection{Automated Task}

Smart hydroponic systems can be classified based on the task that is automated. Therefore, smart hydroponics can be designed to offer assistance to the hydroponic system by maintaining the system's hardware, providing consumables or securing (cyber or physical) the system. Another category helps in crop cultivation by providing the plant with nutrient, lighting or proving cultivation related crop maintenance, like moving seedlings from nursery to hydroponics system, replacing dead plants and harvesting. The final subcategory 


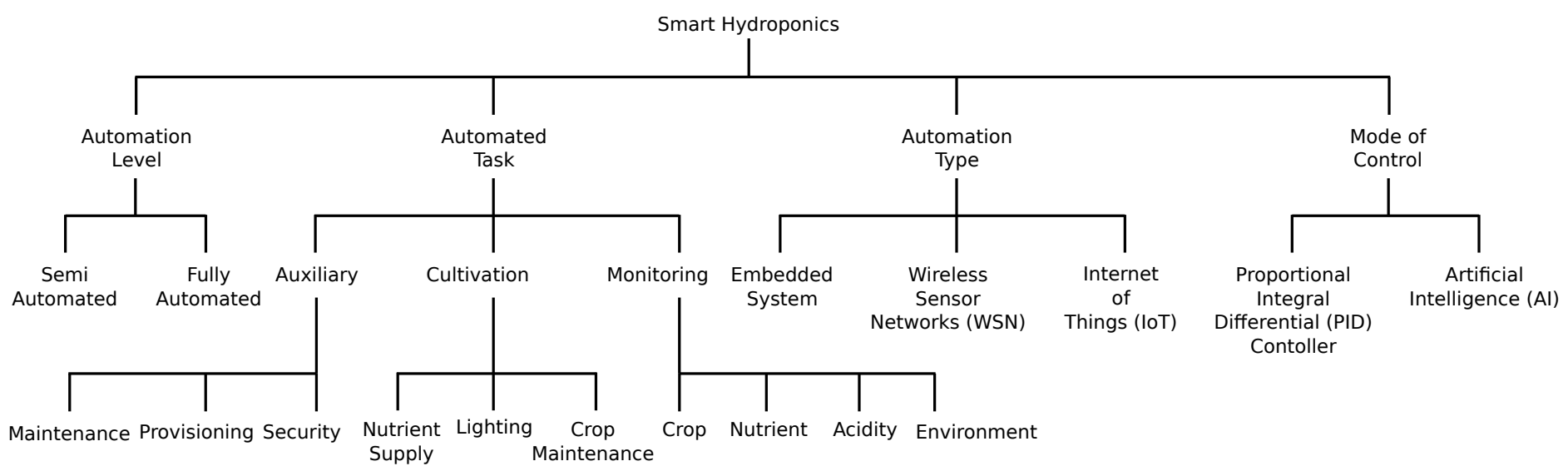

Figure 3: Taxonomy for Smart Hydroponic Systems

monitors some or all aspects of the hydroponic system and reports it to the farmer.

\subsubsection{Auxiliary}

Several smart systems that assist hydroponic systems in crop cultivation have also been proposed in the literature. For example, Tu et al. [29] developed a water harvesting device called Cactus. It is designed to extract water from the air. It can be used in conjunction with IoT to develop a hydroponic system in areas with little or no water. Cactus enable farmers to grow crops in inhospitable areas around the world. It can use solar panels or wind turbines as sources of energy because the system requires a small amount of energy. However, farmers have to account for the latency of the system, since it takes time for the system to condense water from the atmosphere. Therefore, they have to provide enough water to the hydroponic system before the device starts generating its own water.

Saenz et. al [30] developed strawberries collecting a robot for greenhouse hydroponic systems. The proposed system uses artificial vision for identifying the stage of ripeness based on the redness of the strawberries. The system is developed using OpenCV libraries and Arduino Mega for the image processing (sensor), while PUMA manipulator simulated using Solidworks and MatLab for robotic arm (Actuator). The authors focus their automation to harvesting crops rather than their cultivation. The proposed system automates the harvesting of crops, which helps reduce labour cost and the introduction of pests and diseases often brought in by workers into the hydroponic system. The system has shown good performance. It can also be used in harvesting other crops. The system's use of Arduino microcontrollers for image processing may cause an increase in the latency of the proposed system.

Takeuchi [31, 32, 33] pointed out how 3D printed component can be used to develop a hydroponic system and by so doing reducing the cost of hydroponic culture. The paper investigated the use of 3D hydroponic substrate using SBS, PLA, ABS and flexible TPU plastic against the traditional rock wool and sponge. The authors developed custom software that can be used to specify the plantcovered region (in 2D) on the planting substrate. This enables the software to locate places where seeds will be placed. The authors found that the SBS foundation (substrate) holds relatively modest water compare to the traditional hydroponic substrates. The PLA, ABS and flexible TPU perform worse because they do not give way for the root to grow while SBS does. The printed SBS material does not have good water retention (like rock wool), therefore it does not guarantee germination of seeds. This problem is solved by automatically coating the plant seeds in superabsorbent polymer gel (sodium polyacrylate) before placing them in the planting substrate. Due to hardware limitations, not all seeds can be planted, since different seeds have different sizes and shapes. Furthermore, the system has low accuracy in seed placement. Moreover, once the plants germinate they must be irrigated manually.

Sunlight is the most important part of a greenhouse, but dust that accumulates on the house makes it difficult for light to pass through. Cleaning the greenhouse is therefore necessary. Currently, this is done manually, which consumes time and is dangerous for the workers. As such, [34] developed a cleaning root from wood and aluminum frame and a soft-sponge curved wiper blades driven by $12 \mathrm{v}$ DC motor. This makes the robot light-weight and easy to use. The robot is controlled by an MCS-51 microcontroller. Experiments show that the proposed system cleans $85-95 \%$ of the dust on the house at a speed of 1.3 meters per minute. This is an improvement to cleaning systems in the literature who can only work on flats surfaces which is impractical for greenhouses. The system is light-weight therefore less energy is needed to operate it. The paper concludes that the proposed system is low cost and can be used on plastic sheet greenhouses. The system is open-loop, it contains no feedback that indicates the cleanliness of the surface. Therefore and the operator has to be available on-site to overlook it.

Some tasks are difficult to automate using sensors and actuators. Therefore [35] proposed the use of a robot to remotely manage a hydroponic farm. The proposed system uses Microsoft's Kinect v2 for controlling the robot using hand gestures. This allows farmers to manage their farms without any computer training. The proposed system can be used in kick-starting vegetation in remote areas. The authors found that users can carry out activities like lifting, placing, and grasping. However, placing objects in the targeted area is found to be difficult compared to other activities. 


\subsubsection{Cultivation}

The majority of hydroponic systems are developed to help manage the cultivation of crops. Eridani et. al [36] proposed automation of Nutrient Film Technique (NFT) hydroponic system using Arduino Uno as a controller, Total Dissolved Solids (TDS) Sensor for testing nutrient level, a GP2Y0A21 proximity sensor as a water level detector, and servo for replenishing the nutrient from a container. The authors use the electric conductivity (EC) sensor as a TDS sensor. However, it was calibrated using a TDS meter. The author derived a polynomial relating the reading from the TDS meter to the TDS sensor values. The use of the TDS sensor is cheaper than the TDS meter. The proposed system supply more nutrients when the solution is below $800 \mathrm{ppm}$. Also, the TDS sensor is up to $97.8 \%$ accurate. The system controls the amount of nutrient solution in the tank rather than the concentration. Hence, the system may supply more nutrients than the plants needed.

A DIY sensor-based automatic control mobile application for hydroponics is developed in [37, 38]. It is a cheaper and more affordable way of developing small scale hydroponic systems. The system monitors the ambient temperature, water temperature, humidity, light intensity, $\mathrm{pH}$ and EC. The system then uses a set of rules to control the water chiller, LED, light shade, fog spray, and fan in order to manage the environment of the hydroponics. Status of the system is stored in the cloud and the users are allowed to monitor the system through a mobile application. The mobile application also allows farmers to record their yield and a reminder to notify farmers on harvesting date. In a similar experiment is developed by [39]. However, there are some modifications: 1) The hydroponics system is enclosed in a greenhouse, 2) sprinklers are used to control the ambient temperature of the hydroponic system but not the solution, and 3) there are no LEDs to control the lighting of the system. The authors recorded poor performance compared to traditional farming. This shows that the factors selected by [37 matter more than those selected in [39].

Dbritto et. al [40] developed an automated hydroponic system for growing Tomato F1 Hybrid Suhyana seeds. The system is an indoor hydroponic system where amount of water, light, nutrients, and temperature are controlled using Raspberry Pi 3 model B. The authors classify readings from the sensor as follows; Temperature Levels (Cold, Warm, Hot), Water Levels (Low, Normal, High), pH levels (Acid, Neutral, Alkaline) and Light Intensity Levels (Low, Normal, High). They argue that categorizing the reading will make it easier for researchers to develop a model to automate a system whose output sill simply be appropriate or inappropriate. The paper concluded that their taxonomy system can be used for an AI-controlled hydroponic system. However, classification of data to universal categories may be difficult since each plant has a different requirement which may not necessarily fall in the range of other plants.

Arif et al. [41], argue that plants require different nutrients as they age. Therefore an automated hydroponic system needs to be able to determine the age of a plant in order to ensure accurate nutrient supply. The authors in [42] investigated the performance of 3 classical machine learning classifiers: decision tree, Naive Bayes, Multi-Layer Perceptron; and one type of deep neural network in the detecting the freshness of vegetation harvested from a hydroponic system. The proposed system uses image processing and machine learning technologies to detect fresh and withered vegetables. The experiment shows that the decision tree (J48) model was found to have the best accuracy of $98.12 \%$. This system can be used in harvesting and/or monitoring the health of crops grown in a hydroponic system. Also, [41] develop a system that determines the age of plants from their picture using digital image processing technique. The proposed system uses Raspberry Pi 3, a camera module to acquire and process that information which is then saved in a web server. The system is deployed in an NFT (Nutrient FilmTechnique) hydroponic lettuce farm. The authors used vegetation cover to deduce the age of the plant. Vegetation cover is obtain using pixel count after masking to isolate the plant. The proposed system requires neither training nor dataset. The proposed system is able to predict the age of plants with an accuracy of $80 \%$. However, the correlation between the age of a plant and its vegetation cover must be known.

Namgyel et. al [43] developed a smart hydroponic system with LED lighting technology enabled by the IoT system uses the Wi-Fi module as a mode of data transmission and monitoring. SHT 15 sensor was used to measure air temperature and relative humidity. In order to measure and monitor the nutrient temperature, a waterproof temperature sensor is confined in the nutrient solution tank. ML8511 sensors were used to measure the solar energy and surveillance camera to keep track of the physiological growth of the plants. Germination of lettuce seed was done before the experiment and then the seedlings were moved to the hydroponic system 7 days after. The architecture of the system is then divided into four segments where the first three were irradiated with deep red LEDs $(640-660$ $\mathrm{nm})$, deep royal blue LEDs $(440-450 \mathrm{~nm})$ and combination of deep red LEDs $(640-660 \mathrm{~nm})$ and deep royal blue LEDs $(440-450 \mathrm{~nm})$ with the ratio of (1:1) respectively. Solar radiation was the sole light source in the fourth growing segment. Weatherboard embedded with the system controller unit in order to read and receive data from different sensors and transmit to node MCU with embedded ESP8266 Wi-Fi microprocessor and the data flow from sensors are then uploaded to the server through local Wi-Fi network. The system has the ability to monitor and make data accessible to users in real-time. Results indicate that blue supplementary LED light fostered positive effects on lettuce growth, morphology and pigment content.

\subsubsection{Monitoring}

Tagle et. al [44] developed a data acquisition system for hydroponics farming. A DFRobot Arduino Mega 2560 was used as a microcontroller. The system monitors vital parameters such as air temperature, relative humidity, water temperature, water level, $\mathrm{pH}$ level, and light intensity. Also, the acquired data is used to troubleshoot the system in times of failure. An Arduino data logger was designed to collect data from the sensors after every 5 minutes and then store them in an SD card. A 4X20 LCD module was integrated to allow real-time access to the system's performance. The data gathered by all sensors which were stored in an SD card showed fluctuation in environmental condition occurring in the system in real-time which assured ease in monitoring and areas with problems were easily identified for improvement. The data acquisition system 
showed the performance of the hydroponic tower with respect to the growth factors. After validation, it is found that the average percentage error of each growing condition based on their respective standard instrument are near 5\% which shows that sensors available in the market can be used to accurately monitor a hydroponic system.

Recently, [45] successfully developed a hydroponic system that measures only the temperature of the nutrient solution and water level. The system can be monitored through a smartphone. Authors in [46, 47], developed a system that measures water level, $\mathrm{pH}$, electrical conductivity, water temperature, and relative humidity of the system. The data is sent to a database and farmers are notified of events such as the imbalance of nutrients in the system. The system is only designed to monitor the hydroponic environment. Therefore, the system has no actuators and rely on the farmer to manage events [46]. While [47] can control the $\mathrm{pH}$ level, as well as supply water it harvested from rain to the system.

However, there is more to monitoring $\mathrm{pH}$ using $\mathrm{pH}$ sensor than meets the eye. Many $\mathrm{pH}$ meters need re-calibration as they age. A solution is proposed in [48]. The authors proposed an auto-calibration system for monitoring and controlling the $\mathrm{pH}$ (in addition to temperature and EC). The system consists of a chamber where the $\mathrm{pH}$ meter is placed. A series of pumps are connected to the chamber. Three of the pumps are for calibration where one pumps acid, the other pumps base and the third pump buffer (liquid with $\mathrm{pH} 7$ ). The other pump samples nutrient solution from the hydroponic reservoir to the $\mathrm{pH}$ meter's chamber to testing. The last pump pumps water to the chamber to ensure that the $\mathrm{pH}$ meter is clean between the calibration and the testing cycles and increases water level in the hydroponic reservoir. The system was monitored for fifteen (15) days with the aim to maintain a $\mathrm{pH}$ from 5.5 to 5.7. A mean $\mathrm{pH}$ of 5.65 and standard deviation of 0.058 was recorded when using the system, while a mean $\mathrm{pH}$ of 5.00 and a standard deviation of 0.36 is observed on the control system. Although the system shows a promising performance, the large number of moving parts involved will reduce its reliability.

Social media applications have also been used in monitoring hydroponics. Sisyanto et. al [49] developed hydroponic smart farming that can be monitored online via telegram messenger. The system uses different sensors to automatically monitor the vital parameters and environment of the hydroponic system an post it on Telegram messenger using Raspberry Pi 3. The system allows collaboration between hydroponic farmers. With telegram messenger hydroponic farmers communicate with the group/each other and with the telebot one can request the condition of the environment in a hydroponic system and reply back to the sender.

\subsection{Automation Type}

The type of automation is also very important. In this paper, a category is given for the different types of automation. A smart hydroponic system can be a purely embedded system where automation and data remain in-situ. Users have to be physically on the site to monitor or adjust the settings of the system. Another subcategory is Wireless Sensor Networks (WSN) where a distributed network of an embedded system is controlled by a single base station (also known as gateway) users connected to the gateway can access the information. Finally, the IoT based hydroponic system is basically WSNs connected to the internet to improve the scalability and availability of information to the user/farmer.

\subsubsection{Embedded System based Hydroponic System}

This category presents embedded systems that are mainly made up of electronic sensors, controllers and actuators. Usually, data is saved in a memory device where the user can copy it. Settings and configurations can only be done in-situ. For example, in [50, 51], only $\mathrm{pH}$ was measured. The authors develop an AHS using Arduino Mega 2560 micro-controller and ATLAS and ATC pH sensors [51]. Users would key in the $\mathrm{pH}$ level for the targeted crop and the system will maintain that $\mathrm{pH}$ for the targeted hydroponic system. The system was tested for five (5) days with an average deviation of 0.58 and 0.93 for ATLAS and ATC respectively. However, $\mathrm{pH}$ is only one factor that affects plant growth, therefore, optimal plant growth is not possible. The authors used Deep Water Culture (DWC) which is a hydroponic technique where water containing nutrients are fed directly to the roots of the plant continuously. The system consists of a $\mathrm{pH}$ and water level sensor, an actuator system, using a valve controlled solution mixer and water pump [50]. The proposed system uses a glass electrode $\mathrm{pH}$ sensor which measures both $\mathrm{pH}$ and temperature and feeds it to the microcontroller SK 40C for decision making. The setup ensures reduced energy consumption. The authors establish that there is a need for at least $5.64 \mathrm{ml}$ of acid and alkaline to increase the acidity and alkalinity by $0.312 \mathrm{pH}$ and 0.244 $\mathrm{pH}$ respectively. These levels can be achieved through the use of a feeder valve. The glass electrode meter is prone to errors [52].

More sensors were added in [53, 54], a sensor module capable of measuring EC, water temperature and water level of a hydroponic system. The system uses the capacitance of a cable ribbon to measure the level of the water. It uses a TI TMP20 sensor coated by resin connected at the end of the aforementioned ribbon cable to measure the temperature and electrodes to measure the EC of the nutrient solution. An oscillator is used to measure the capacitance of the cable. The proposed sensor module shows that capacitance and water levels are proportional, which is easy for sensing and analysis. The authors found that the accuracy of the proposed system is within $1 \%$ of commercial sensors. However, they pointed out that the structure of the electrodes will be reviewed because the error in EC increases when the distance between the electrodes changes. The sensor module shows a logarithmic relationship with the capacitance which is difficult to convert to digital at high levels of EC since the change is small.

Velazquez et. al [55] proposed an automated hydroponic system for a cherry tomato. The system ensures that the $\mathrm{pH}$ is maintained between 5.5 to 6 and the electric conductivity (salinity) between 1.8 $\mathrm{mS}$ to $3 \mathrm{mS}$. The system uses PIC16F877A microcontroller and an ion-selective electrode (ISE) is used for $\mathrm{pH}$ measurement while a pair of gold wires separated $1 \mathrm{~cm}$ apart is used for EC sensor. The authors added conditioning circuits and amplifiers to the $\mathrm{pH}$ sensor and the EC sensor. The use of amplifiers and conditioning circuit help reduce the amount of noise in the readings. The results showed high accuracy on $\mathrm{pH}$ measurements with a correlation coefficient of 0.994 for expected values and a standard deviation of 0.01 after repeated measurements. The use of extra circuitry will increase 
energy consumption and latency of the proposed system.

A semi-automatic hydroponic system that controls the humidity, ambient temperature and $\mathrm{pH}$ is developed in [56]. The system switches between automatic monitoring and manual control. This allows the system to maintain optimal conditions for the plant regardless of whether the user is connected or not. Users/farmers can also monitor the system through an LCD screen on site. For more flexibility, Nalwade and Mote [57] developed an automated hydroponic system that ensures the potential of Hydrogen $(\mathrm{pH})$ and Electric Conductivity (EC) of nutrients is maintained by the system and measured automatically by sending the information to the farmer through GSM. Aeration problem is solved using an oxygen pump. The energy problem solved using solar panels. Similarly, [58] proposed a cost-effective and environmentally sound automated solar-powered aquaponics system, for local communities. It consists of a water circulation system that circulates water to an aquaculture tank and hydroponic beds, aquaponics control and monitoring system actuators, GSM shield and NI LabVIEW, solar energy conversion system, cooling, and heating systems that maintain the air and water temperature. However, the nutrient level from the fish tank is not monitored. This may lead to a high concentration of nutrients supplied to the plant.

\subsubsection{Wireless Sensor Networks (WSN)}

Wireless Sensor Network (WSN) is the network of computing devices with limited resources (such as processor, memory, energy) aimed at sensing their environment [59,60,61]. WSN finds application in health-care, military, manufacturing industry and agriculture [62, 63, 64, 65].

Figure 4 shows a typical WSN. It consists of: Sensor nodes who are responsible for sensing the environment, Relay nodes are responsible for forwarding information to the sink node. Relay nodes are needed because nodes in WSN are short-range devices. Sink nodes connect the sensor network with the outside world usually a workstation. Actuator nodes are responsible for manipulating the environment; such as turning on/off valves, finally, the user (workstation) is responsible for collecting and analyzing data from the network. In this research, WSN will be used to automate the hydroponic system in order to enable hydroponic farming easier and cost-effective.

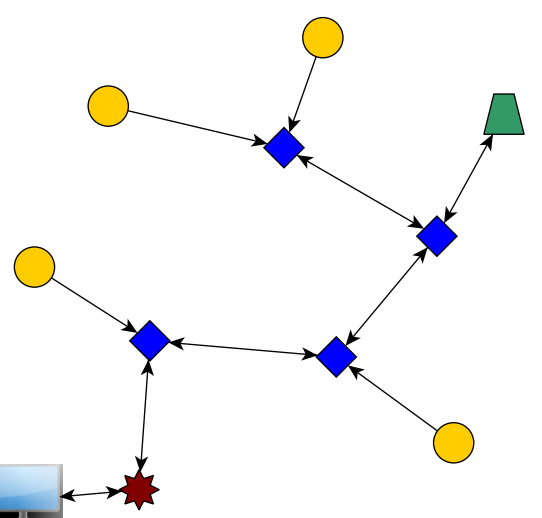

Sensor Nodes

Relay Nodes

Sink Nodes

Actuator Nodes

User

Figure 4: A typical Wireless Sensor Network (WSN).
Jai et. al [66] proposed a system consisting of soil moisture, $\mathrm{pH}$ level, light intensity, water temperature, humidity and temperature sensor. The sensors forward their data to the control unit which is made up of Arduino Uno microcontroller. A predefined threshold is set for the moisture sensor. When the level is below a certain threshold the microcontroller increases the amount of water circulating in the system. The soil moisture sensors are placed in every pot of the system to ensure proper nutrient supply. Since all nodes are individually monitored to ensure adequate water and nutrient supply. The system ensures no water wastage and that the plants got adequate water and nutrient supply. The system uses a predetermined threshold and the water level is compensated proportionally. This leads to oscillation in the system.

When using WSN for hydroponics there is need to choose a suitable transmission protocol that will ensure low energy consumption and low latency in communication. The aforementioned factors are the most important because most smart hydroponic systems transmit only commands and state of the system. As such, researchers use zigbee transmission protocol. In [67], zigbee stack is used to develop a real-time smart hydroponic system. The authors found that although the performance of the system is satisfactory, the distance of the system from a the control station is inversely-proportionally affecting the performance of the system. In addition, the authors cite size of memory of the sensor node as a challenge to the efficiency of the system.

\subsubsection{Internet of Things (IoT) based Hydroponic System}

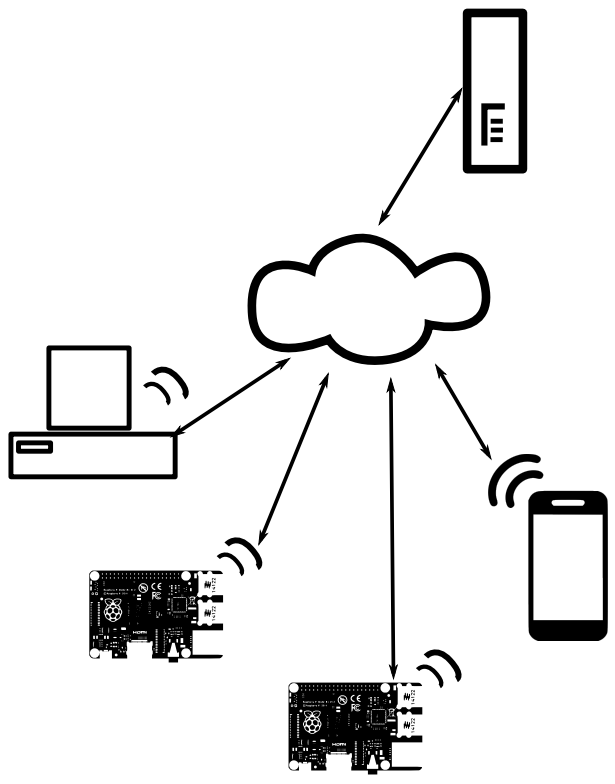

Figure 5: A typical Internet of Things (IoT) setup.

As shown in Figure 5, IoT is a network of embedded systems, smartphones and personal computers that communicate through the internet. Data generated from IoT systems are saved and monitored through a server or a broker depending on the design. Researches have been carried out on the use of IoT in hydroponic systems [68]. IoT based hydroponics system is designed to monitor and/or control a hydroponic system with the help of a server. The system commu- 
nicates with the server through Ethernet, Wi-Fi, GPRS or satellite radio communication.

Fernandes et al. [69] developed an Arduino and Raspberry Pibased solution which are connected with USB cable and Pi card memory to save the experimental data. The system was able to allow external users and programmers to connect to the Pi via the internet. The system was able to control the temperature and humidity of the greenhouse based hydroponic system. The system improves the efficiency of the growth of crops. The system was proven to be much faster compared with traditional techniques. However, the configuration of the system was not easy due to the extra plug-ins and third-party software required. Also, the system allows the monitoring of plant growth by applying Image Processing. unfortunately, the system was designed to favor crop production in the summertime only, since it provides only cooling systems to control High temperature. Furthermore, neither the $\mathrm{pH}$ or Nutrient solution concentration (EC) levels were measured so, the information obtained from the system regarding nutrient combination and $\mathrm{pH}$ will not be reliable.

Siregar et al. [70] proposed a closed hydroponic monitoring system designed with PH sensor, Electric Conductivity Sensor, water temperature sensor, air temperature, Light Sensor, GSM / GPRS, Open Garden Shield, Open Garden Hydroponic, and Arduino Uno microcontroller. The system was used to test lettuce, red spinach, and mustard Pak Choy plants. All sensors in the proposed system are combined using Open Garden Shield and Open Garden Hydroponics. The open garden shield is an open-source Arduino shield that is used in automating gardens. The data received by the shield from the sensors is sent to the Arduino Uno using I2C communication. Finally, the data is sent to a remote server with the help of a GSM shield. The proposed system used the open garden shield and open garden hydroponics which makes the reproduction of results easy. The authors conclude that the lettuce, red spinach, and mustard Pak Choy plants perform well. The proposed system has no actuators to control the environment, it depends on the farmer for control.

The authors in [71] developed an automated aquaponic system with minimum resource requirements. The system automatically feeds the fish and water the plants with the help of the GSM network The proposed system consists of two (2) modules; Automatic water supply and Automatic fish food feeder. The earlier module is responsible for supplying the plants with nutrient water from the aquarium while monitoring the water level of the aquarium. Whenever the level of water in the aquarium goes down, the module replenishes it from a secondary tank. The second module is responsible for feeding the fish. Furthermore, the system also has heater and temperature sensors for controlling the temperature of the water in the fish tank. It also controls the lighting provided by Light Emitting Diodes (LEDs) to ensure photosynthesis. The proposed system is portable, therefore it can be installed at home or office. Furthermore, the system is made up of a few components therefore is it cheap to develop. The authors observe that the proposed system consumes only $3.87 \mathrm{~L}$ of water in two weeks. Also, the fish consume 5-10 gm of food in two days. It is also found that an automated aquaponic system with an aquarium and plant bed is feasible. The proposed system lacks acidity and basicity control system. Also, no system is provided to ensure the well being of the fish.
In [72] developed a small scale intelligent plant care hydroponic box using IoTtalk [73]. The system allows users to easily add, remove or exchange sensors and actuators in the plant-care system and it also provides environment driven control methods between the sensors and actuators. In order to make analysis, the sensors generate environment data and send it to a server through Arduino Yun via wi-fi, then data processing system executes the environment driven controls function based on the user-defined threshold values and finally the IoTtalk plant-care intelligence directs the actuators in the response system to take action. Similar IoTtalk-based hydroponic systems have been proposed in [74, 75]. ThingSpeak, like IoTtalk is an IoT platform [76]. Lakshmiprabha et. al [77] used ThingSpeak to manage and monitor water level, humidity and temperature with the help of Rasberry Pi 3 B + and Field-Programmable Gate Array (FPGA). Also, a larger model of the system is developed by in [78, 79]. The system allows for modularity, thereby enabling ease of maintainability. However, the system relies on communication with a server that may not be available all the time. Also, a fixed threshold may not be optimal since the needs and consumption rate of the plants' changes as they grow.

An IoT based smart hydroponic system is presented in [80] where the sensor communicates with the server through the Ethernet. The authors developed a closed hydroponic control and monitoring system with the help of Arduino Uno, a clock module (RTC I2C), a relative humidity and temperature sensor (DHT11), a water pump (HJ 701), a fan (POLUX PH 2009) and an electrical resistance and an aerator (AC 500) was also used to oxygenate the water. An Ethernet module is used to send readings of the different sensors to a server. The system is developed for the Stevia plant. Also, aeration and lighting are accurately controlled with the help of the RTC clock. The proposed system is cheaper than the most commercially available hydroponic system. The authors of the proposed system were able to save to $76 \%$ of the cost price of a commercially available AeroFlo hydroponic system.

IoT based hydroponic systems are known to have many interconnected devices, as well as software. As such [81, 82] proposed a cloud-based IoT for controlling and monitoring hydroponic systems using publish-subscribe middleware over MQTT protocol. The use of middleware allows the network administrator to set the quality of service for the system which will help guarantee delivery even if the network itself is not reliable. The authors in IoT based hydroponic systems are known to have many interconnected devices, as well as software. As such [81] found that the response time of the system is 7.21 seconds. Also, they found that the system needs at least 21.55 seconds to determine if a node is offline. Authors in [83] integrated TLS/SSL to encrypt MQTT packets. Moreover, the authors used Raspberry Pi as the broker/server, where the Ardunio (client) sends its data including the image of the plants. The remote management system was developed using open source libraries.

To solve the volume and velocity problem of data developed in a hydroponic system, data fusion is used in [84]. Data fusion is a technique where information from different sensors is combined (fuse) to achieve a more accurate result. Data fusion also reduces the transmissions in the network thereby reducing traffic and energy consumption. The authors proposed an automatic control and management system for tropical hydroponic cultivation. The system monitors the power status, temperature, humidity, water 
level, $\mathrm{pH}$, and $\mathrm{EC}$ data which are sent to a remote database over the internet. Users can access the status of the hydroponic sensor through a mobile application. The system is a two-tier network containing sensors $\left(S_{i}\right)$ and data fusion sensors $\left(D F_{i}\right)$. The sensors forward their sensed data to the data fusion sensors where the data is combined to achieve a more accurate result before saving it in the remote database. The authors used data fusion techniques to improve the accuracy of data received from the sensors. This is achieved by fusing information from the ultrasonic sensor with a solenoid valve to get water level and SHT31 sensor with a foggy solenoid valve for temperature and humidity control. The system also notifies the user of a power outage. The system is able to improve the accuracy of readings from the sensor nodes without either taking several readings or using many different sensors.

Another solution is the use of edge computing. Edge computing is an IoT paradigm where processing devices (with communication capabilities) are placed at the edge of the network close to the user, to provide services to the user in lieu of the server [85]. The author in [86] argue that edge computing is the cost effective way of ensuring connectivity in smart hydroponics. In their paper, the authors proposed a three-tier system with local, edge and cloud layer. The local layer is responsible for monitoring the crops and carrying out atomic action on them at real-time. The edge layer is responsible for monitoring and managing the hydroponic system, while the cloud is responsible for data collection and analysis. The system uses FIWARE, ORION Context Broker [87] over MQTT to allow the edge and the cloud layers to communication efficiently. The edge layer uses Network functions virtualization (NFV), which increases it flexibility. The system reduce water consumption by $30 \%$ and some nutrients by $80 \%$.

\subsection{Mode of Control}

In smart hydroponic systems where actuators are involved, controlling how the actuators behave is a very important aspect of the system. In this paper, mode of control is categorized into Proportional Integral and Derivative (PID) controller or Artificial Intelligence (AI). The PID controllers are the type of controllers developed using control engineering techniques they are widely used because they are flexible and easy to develop [88]. AI can also be used to control how the actuators perform their actions to ensure they do not damage the system or themselves. In this paper, a broader interpretation of the word AI is used; any algorithm that can perform human tasks is placed in this category.

\subsubsection{Proportional Integral Derivative (PID) Controller}

Proportional, Integral and Derivative (PID) controller is a control system that uses feedback to adjust the output to the desired level [89]. PID controllers are popular because they are easy to develop and tunning, wide availability and high success rest.

Asumadu et. al [90] proposed a computer-based hydroponic control system using LABVIEW. The system measures the $\mathrm{pH}$ level using EC and the water level using an ultrasonic sensor. The control system adjusts the water level in the system by observing the error between the current level and the predetermined level. The use of a computer and LABVIEW improves the precision of the height. Also, the experiment shows that a hydroponic setup can be controlled through an instrumentation software.

However, since the system is a proportional controller $\mathrm{pH}$ level will oscillate. The work carried out in [91] is a testament to that fact. The authors developed an automated hydroponic system for green mustered. The system ensures that the plants are kept in a deep water culture (DWC) of pH between 6.0-7.5, which is set by a keypad and managed by Arduino Mega 2560 microcontroller. The authors pointed out that there is a delay in the $\mathrm{pH}$ level change after more fertilizer is added. Therefore, they used two tanks (main tank and hydroponic tank), the main tank is used to verify the $\mathrm{pH}$ level of the water before is it finally pumped to the hydroponic tank where is it used for growing the plants. The use of a separate tank allows the authors to stir the liquid and observe its response to the added nutrients. The authors found that after a certain time (16 days) the system begins to oscillate. They also pointed out that the reading accuracy of the $\mathrm{pH}$ meter used has a major role in the performance of the system.

To reduce oscillations encountered in a proportional controller, Adhau et. al [92] developed an automated closed hydroponic system with proportional integral and derivative (PID) controller is developed with the help of AVR microcontroller and LabVIEW. The system uses DHT11 humidity sensor, LM 325 temperature sensor, electrodes for $\mathrm{pH}$, and ambient light sensors, while it uses fans, blowers, halogen light bulbs, pumps and motors as actuators to control the state of the system. The authors used a PID controller which allows their system to control the environmental parameters with little or no oscillation. The PID is tuned using Zeigler-Nicholas closed-loop tuning methods. The authors also used the AVR microcontroller rather than the traditional LabIEW's data acquisition (DAQ) card, which reduces the cost of implementing the system. The proposed system improves the response time of the controller with minimal oscillation. The system shows great stability and smooth control actions due to the use of a PID controller. The requirements of a hydroponic system change as the plants grow, however, the proposed system uses fixed values which require human interference in order to perform efficiently.

\subsubsection{Artificial Intelligence (AI) based Hydroponic System}

Gartphol et. al [93] developed predictive models for Lettuce quality from the Internet of Things-based hydroponic farms. The authors developed a smart hydroponic lettuce farm using the Internet of Things to gather environmental data and control the farm's operation in real-time. A large dataset was generated at the end of the experiment which was used to create regression models using machine learning techniques. Some of the features used were environmental data such as the amount and intensity of light, humidity, temperature, together with weekly measurements of plant growth. Internet of Things allows automatic operation which can be accessed within a household or office area. A good learning result has not been achieved by the model due to errors such as those found in measuring the lettuce's height because of changes caused by blowing wind which causes the crop to sway. Other errors are those found from the stem's width. In [94], Bayesian Network is reported to be $84.53 \%$ accuracy and a $66.67 \%$ more yielded crops than the manual system.

Fuzzy logic has been used to solve a variety of problems in an 
automated hydroponic system [95, 96]. Mashumah et. al [95] developed a Nutrient Film Technique (NFT) hydroponics that uses fuzzy logic control to set the threshold value for the electrical conductivity (EC) level. The system uses a webcam Logitech B525 camera to capture the image of a plant and converts it to HSV Histogram to provide EC setpoint value. An ultrasonic distance sensor HC-SR04 measures the volume of the water with an error rate of $15.6 \%$. The saturation value of the leaves is used to determine the set point of EC value for the control of the system. The saturation value is chosen because of its high level of linearity with the age of the plant. EC sensor SKU:DRF0300 measures the concentration of the nutrient in the water. It then uses EC error and water volume as inputs of fuzzy logic to adjust the opening time of the nutrients and water valves. The system can provide and retain the EC value given according to the age of the plant with an error rate of $8.9 \%$. In another research, scientists investigate the most suitable colour space for vegetation image processing in smart hydroponics [97]. The authors [97] show that the HSV method has an average accuracy of $75.33 \%$ while the CIELAB method has a greater average accuracy of $78.39 \%$. Research conducted in [98, 96] shows that the steady-state time (i.e. time to reach the targeted value) of fuzzy logic-based EC controller is around $110 \mathrm{sec}$, which is quite slow.

Also, researchers in [99] developed a novel fuzzy logic-based control system combined with a genetic algorithm (GA) to maintain the $\mathrm{pH}$ level of a hydroponic system. The authors argue that a fuzzy logic control (FLC) system is more suitable than PID controllers because PID controllers suffer limited feedback. The system has five storage tanks: acid, base, buffer, nutrient A and nutrient B. The tanks are controlled by valves in order to maintain the $\mathrm{pH}$ level. A drain valve is used to control the level of mixture in the hydroponic tank. The nutrient solution is monitored using a $\mathrm{pH}$ meter and EC meter. The authors used 199 fuzzy rules obtained from experimental observations and expert opinions from researchers in MCRC, India. The authors developed a novel Mamdani fuzzy inference system (FIS), which uses a given set of control parameters to grade the quality of a hydroponic solution. The result is then fed to a GA as a fitness function in order to obtain the optimal valve for the control signals. The authors pointed out that their proposed FIS performs better than fuzzy or PID controllers. The proposed algorithm proved efficient in terms of convergence rate and resource utilization compared to conventional error based algorithms used in solution control. However, the use of a drain valve in the proposed system will lead to wastage.

The authors in [100] grew wheat (Triticum aestivum L.) in glass housed hydroponic environment in order to classify nutrient deficiency using multilayer perceptron (MLP) neural network with using hyperspectral data as input. The authors create a nutrient mixture with the investigated nutrient absent. Then spectral reflectance of the leaves in wavelengths $401 \mathrm{~nm}$ to $770 \mathrm{~nm}$ is fed to the 3layer (input, hidden, and output) MLP. The aim of the research is to investigate the performance of MLP in classifying leaves with a deficiency in deficient in nitrogen $(\mathrm{N})$, phosphorus $(\mathrm{P})$, potassium $(\mathrm{K})$, and calcium $(\mathrm{Ca})$. This research can be used to automate the fertilizer application process in hydroponic systems. The authors show that MLP with backpropagation algorithm used for classification performs (N 90.9\%, P 100\%, K 90\%, and Ca 100\%) better than regression model (N 93.0\%, P 87.2\%, K 91.9\%, and Ca 97.4\%).
Although the Artificial Neural Network (ANN) shows excellent performance, training data set for other crops and vegetables may not be easy to come by.

Furthermore, [101] proposed an ANN technique for controlling the light intensity by observing the net photosynthesis of the plants in a hydroponic system. The net photosynthesis is measured using the Koito model KMC series assimilation chamber and $\mathrm{C02}$ analyzer, while the light intensity is measured using the LI-COR model LI-185B quantum sensor. The light intensity and net photosynthesis were measured at an interval of 2 minutes from 8 o'clock to 11 o'clock. This data is then fed it to the neural network for training. The ANN uses backpropagation for training. The proposed system is self-adaptive since it can learn from any given environment. The authors found that there is no significant difference between the 3-layer and 4-layer ANN. Also, the authors compared a 3-layer ANN, 3-layer recurrent ANN, Group Method of Data Handling (GMDH), and least mean square techniques. It is found that there is no significant difference in the performance of the first three techniques, while the last-mentioned technique is performed the worse of them. Single feature (light intensity) was used in measuring the performance of the proposed system which is not enough to account for the net photosynthesis of the hydroponic system.

More dimension was added to the ANN in [102]. Here, the authors developed a hybrid 4-stage control system using neural networks and genetic algorithm. The system's objective is to maximize the total leaf length to stem diameter (TLL/SD), which is an indicator of a healthy plant. The neural network is used to identify the environmental factors that affect the plant's physiology (i.e. TLL/SD). Then the genetic algorithm is used to control them. The 3-layer neural networks take nutrient concentration (NC) and light intensity as input and TLL-SD ratio as output. It was trained using the backpropagation technique. The neural network is used to obtain fitness functions for the nutrient concentration at 4 stages. These fitness functions at then used to obtain the nutrient concentrations at the four stages $\left(N C_{1}, N C_{2}, N C_{3}\right.$ and $\left.N C_{4}\right)$. The proposed system uses two AI techniques to observe the environment and to detect the right nutrient concentration for the plant at different stages of its life. Therefore, the system is adaptive to the environment and the type of plant. The proposed system shows that the NC should be a little high in the first stage of the plants' growth, then a little low in the second stage, then a little higher than the first stage in the third stage and a little high than the third stage in the fourth stage. The system was observed for 24 days and the TLL-SD ratio shows an increase of $\approx 9.375$ per day. The proposed system needs a dataset which is hard to come by.

\section{Challenges in Automating Hydroponic Systems}

So far, researchers have made a tremendous contribution to the development of efficient smart hydroponic systems. However, several challenges in this area are still untouched. Some of these challenges are:

1. Security of the system is a very important problem. For companies and countries to cozy up to the smart hydroponic 
Table 1: Summary of the Automated Hydroponic Systems

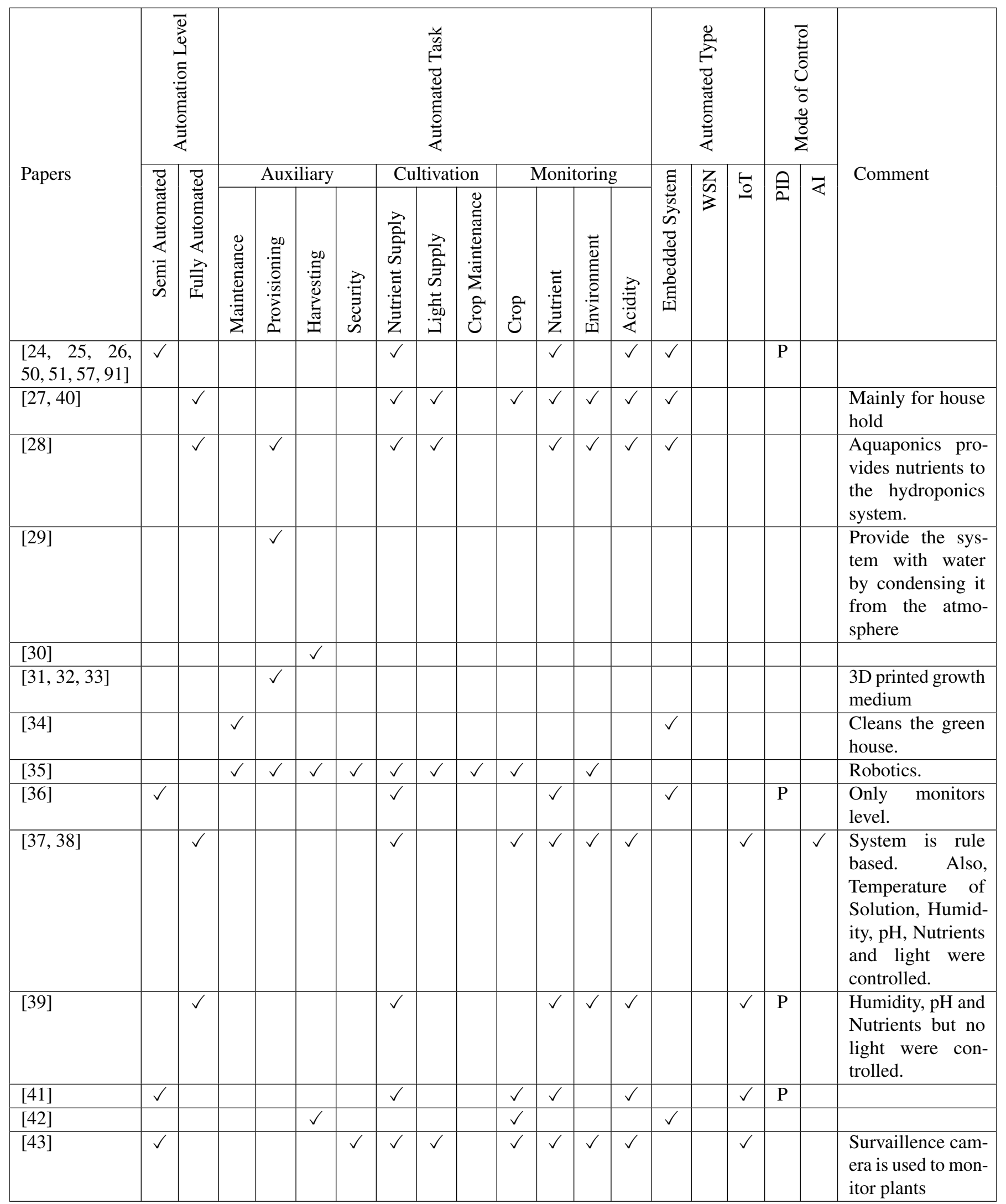


Table 2: Summary of the Automated Hydroponic Systems Continued

\begin{tabular}{|c|c|c|c|c|c|c|c|c|c|c|c|c|c|c|c|c|c|c|c|}
\hline \multirow{3}{*}{ Papers } & \multicolumn{2}{|c|}{ 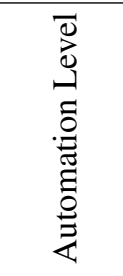 } & \multicolumn{11}{|c|}{ 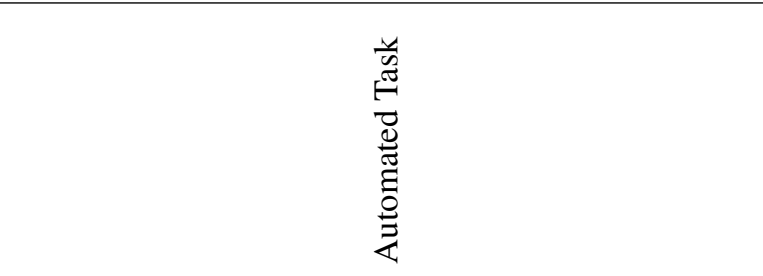 } & \multicolumn{3}{|c|}{ 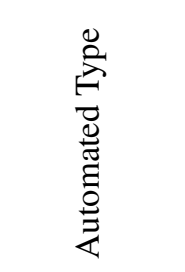 } & \multicolumn{2}{|c|}{ 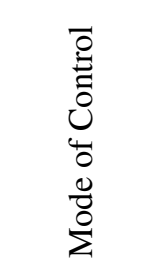 } & \multirow{3}{*}{ Comment } \\
\hline & \multirow[b]{2}{*}{ 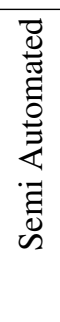 } & \multirow[b]{2}{*}{ 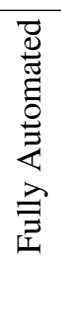 } & \multicolumn{4}{|c|}{ Auxiliary } & \multicolumn{3}{|c|}{ Cultivation } & \multicolumn{4}{|c|}{ Monitoring } & \multirow[b]{2}{*}{ 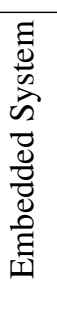 } & \multirow{2}{*}{$\begin{array}{l}Z \\
n \\
3\end{array}$} & \multirow{2}{*}{$\stackrel{0}{\circ}$} & \multirow{2}{*}{ 空 } & \multirow{2}{*}{ 飞 } & \\
\hline & & & 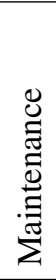 & 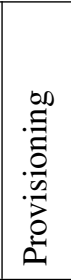 & 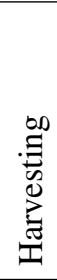 & 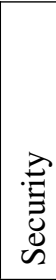 & 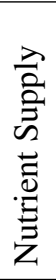 & 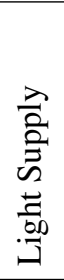 & 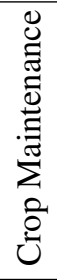 & 气̊? & 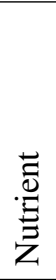 & 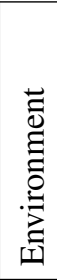 & $\stackrel{\substack{0 \\
\frac{0}{2}}}{4}$ & & & & & & \\
\hline [44] & & & $\checkmark$ & & & & & & & & $\checkmark$ & $\checkmark$ & $\checkmark$ & $\checkmark$ & & & & & $\begin{array}{l}\text { used for trou- } \\
\text { bleshooting } \\
\text { hydroponic } \\
\text { system }\end{array}$ \\
\hline [45] & $\checkmark$ & & & & & & $\checkmark$ & & & & $\checkmark$ & $\checkmark$ & & & & $\checkmark$ & $\mathrm{P}$ & & \\
\hline [46] & & & & & & & & & & & $\checkmark$ & $\checkmark$ & $\checkmark$ & & & $\checkmark$ & & & \\
\hline 477 & $\checkmark$ & & & $\checkmark$ & & & $\checkmark$ & & & $\checkmark$ & $\checkmark$ & $\checkmark$ & $\checkmark$ & $\checkmark$ & & & $\mathrm{P}$ & & \\
\hline [48] & $\checkmark$ & & & & & & & & & & & $\checkmark$ & & $\checkmark$ & & $\checkmark$ & & & \\
\hline [49] & & & & & & & & & & $\checkmark$ & $\checkmark$ & $\checkmark$ & $\checkmark$ & & & $\checkmark$ & & & $\begin{array}{l}\text { System uses so- } \\
\text { cial media }\end{array}$ \\
\hline [53, 54,56$]$ & $\checkmark$ & & & & & & $\checkmark$ & & & & $\checkmark$ & $\checkmark$ & $\checkmark$ & $\checkmark$ & & & $\mathrm{P}$ & & \\
\hline$[55]$ & & & & & & & & & & & $\checkmark$ & & $\checkmark$ & $\checkmark$ & & & & & \\
\hline [58] & & $\checkmark$ & & $\checkmark$ & & & $\checkmark$ & $\checkmark$ & & & & $\checkmark$ & & & $\checkmark$ & & & & $\begin{array}{l}\text { Nutrient is pro- } \\
\text { vided by the } \\
\text { aquaponic system }\end{array}$ \\
\hline [66] & $\checkmark$ & & & & & & $\checkmark$ & & & $\checkmark$ & $\checkmark$ & $\checkmark$ & $\checkmark$ & & $\checkmark$ & & & & \\
\hline 67] & $\checkmark$ & & & & & & $\checkmark$ & & & & $\checkmark$ & $\checkmark$ & $\checkmark$ & $\checkmark$ & $\checkmark$ & & & & \\
\hline 699 & $\checkmark$ & & & & & & $\checkmark$ & & & $\checkmark$ & & & & & & $\checkmark$ & $\mathrm{P}$ & & $\begin{array}{l}\text { Temperature con- } \\
\text { trol system }\end{array}$ \\
\hline [70] & & & & & & & & & & & $\checkmark$ & $\checkmark$ & $\checkmark$ & & & $\checkmark$ & & & \\
\hline \begin{tabular}{|l|l|}
71,72, & 74, \\
$75,78,79 !$ &
\end{tabular} & & $\checkmark$ & & & & & $\checkmark$ & & & & $\checkmark$ & $\checkmark$ & $\checkmark$ & & & $\checkmark$ & $\mathrm{P}$ & & \\
\hline$[77,84$ & $\checkmark$ & & & & & & $\checkmark$ & & & & $\checkmark$ & $\checkmark$ & $\checkmark$ & & & $\checkmark$ & $\mathrm{P}$ & & \\
\hline [80] & & $\checkmark$ & & & & & $\checkmark$ & $\checkmark$ & & & $\checkmark$ & $\checkmark$ & $\checkmark$ & $\checkmark$ & & $\checkmark$ & & & \\
\hline$[81,82, ?]$ & & $\checkmark$ & & & & & $\checkmark$ & & & & $\checkmark$ & $\checkmark$ & $\checkmark$ & & & $\checkmark$ & $\mathrm{P}$ & & $\begin{array}{l}\text { Middleware is } \\
\text { used to help with } \\
\text { heterogeneity }\end{array}$ \\
\hline [83] & & $\checkmark$ & & & & $\checkmark$ & $\checkmark$ & & & $\checkmark$ & $\checkmark$ & $\checkmark$ & $\checkmark$ & & & $\checkmark$ & $\mathrm{P}$ & & \\
\hline [86] & & $\checkmark$ & & & & & $\checkmark$ & $\checkmark$ & & & $\checkmark$ & $\checkmark$ & $\checkmark$ & $\checkmark$ & & $\checkmark$ & & & $\begin{array}{l}\text { Edge computing } \\
\text { is used to reduce } \\
\text { latency }\end{array}$ \\
\hline [90] & $\checkmark$ & & & & & & $\checkmark$ & & & & $\checkmark$ & & $\checkmark$ & & & & $\mathrm{P}$ & & $\begin{array}{l}\text { Computer is used } \\
\text { for automation }\end{array}$ \\
\hline [91] & $\checkmark$ & & & & & & $\checkmark$ & & & & $\checkmark$ & $\checkmark$ & & $\checkmark$ & & & $\mathrm{P}$ & & \\
\hline [92] & & $\checkmark$ & & & & & $\checkmark$ & $\checkmark$ & & & $\checkmark$ & $\checkmark$ & $\checkmark$ & & $\checkmark$ & & PID & & \\
\hline$[93,94$ & & $\checkmark$ & & & & & $\checkmark$ & & & $\checkmark$ & $\checkmark$ & $\checkmark$ & $\checkmark$ & & & $\checkmark$ & & $\checkmark$ & \\
\hline [95] & $\checkmark$ & & & & & & $\checkmark$ & & & $\checkmark$ & $\checkmark$ & & $\checkmark$ & & $\checkmark$ & & & & \\
\hline [98, 96] & $\checkmark$ & & & & & & $\checkmark$ & & & & $\checkmark$ & & $\checkmark$ & & $\checkmark$ & & & $\checkmark$ & Fuzzy logic only \\
\hline
\end{tabular}


Table 3: Summary of the Automated Hydroponic Systems Continued

\begin{tabular}{|c|c|c|c|c|c|c|c|c|c|c|c|c|c|c|c|c|c|c|c|}
\hline \multirow{3}{*}{ Papers } & \multicolumn{2}{|c|}{ 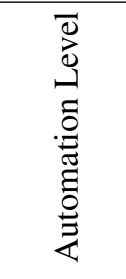 } & \multicolumn{11}{|c|}{ 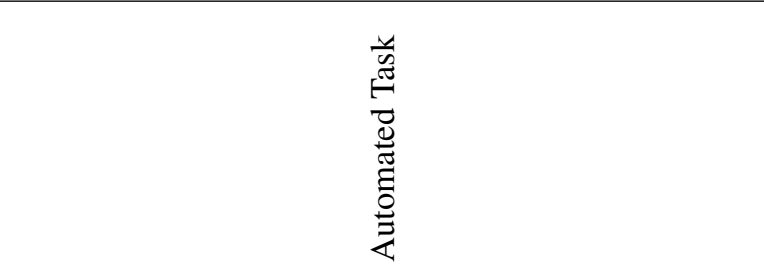 } & \multicolumn{3}{|c|}{ 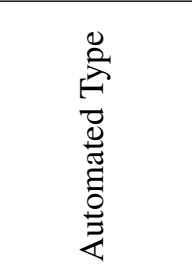 } & \multicolumn{2}{|c|}{ 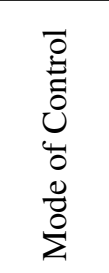 } & \multirow{3}{*}{ Comment } \\
\hline & \multirow[b]{2}{*}{ 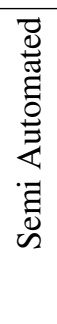 } & \multirow[b]{2}{*}{ 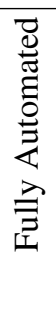 } & \multicolumn{4}{|c|}{ Auxiliary } & \multicolumn{3}{|c|}{ Cultivation } & \multicolumn{4}{|c|}{ Monitoring } & \multirow[b]{2}{*}{ 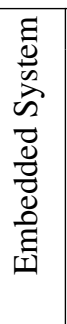 } & \multirow{2}{*}{$\begin{array}{l}Z \\
Z \\
3 \\
3\end{array}$} & \multirow{2}{*}{ 흥 } & \multirow{2}{*}{$\hat{\mathbf{2}}$} & \multirow{2}{*}{ 元 } & \\
\hline & & & 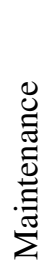 & $\begin{array}{l}.0 \\
.0 \\
.0 \\
.0 \\
0 \\
0 \\
0 \\
0\end{array}$ & 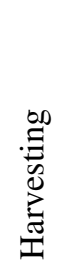 & 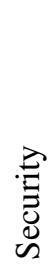 & 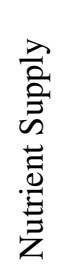 & 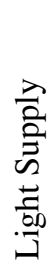 & 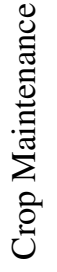 & ఫे & 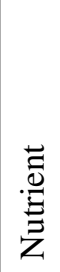 & 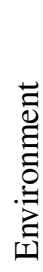 & 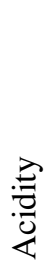 & & & & & & \\
\hline [99] & $\checkmark$ & & & & & & $\checkmark$ & & & & $\checkmark$ & & $\checkmark$ & & $\checkmark$ & & & $\checkmark$ & $\begin{array}{l}\text { Fuzzy logic com- } \\
\text { bined with Ge- } \\
\text { netic algorithm to } \\
\text { control pH }\end{array}$ \\
\hline$[100,101]$ & $\checkmark$ & & & & & & & $\checkmark$ & & & & $\bar{\checkmark}$ & & $\checkmark$ & & & & $\checkmark$ & $\begin{array}{l}\text { Computer directly } \\
\text { reads the sensors. } \\
\text { ANN is used for } \\
\text { AI. }\end{array}$ \\
\hline [102] & $\checkmark$ & & & & & & $\checkmark$ & $\checkmark$ & & & $\checkmark$ & $\checkmark$ & & & & & & $\checkmark$ & $\begin{array}{l}\text { ANN is used for } \\
\text { AI. }\end{array}$ \\
\hline
\end{tabular}

system they need to be certain that their system is secured from intruders.

2. Automation of harvesting in hydroponics with the help of IoT has not been explored. Since plants are grown in batches, the manual harvesting method may lead to the infection of the younger crops with human beings as vectors. Furthermore, since the automated system can work tirelessly, it is more economical to use smart systems in the harvesting of the crops.

3. Impact of the sensors used in the development of plants has not been investigated. For example, what is the effect of electricity on plants?

4. Further investigation should be made into the tolerance plants to high EC and/or $\mathrm{pH}$. The knowledge of how tolerant plants are at extreme levels of $\mathrm{pH}$ will allow system designers to develop systems that can optimally manage the hydroponic systems.

5. The use of controllers should also be investigated. Stress on fish (in aquaponics) and plants due to fluctuations in EC and $\mathrm{pH}$ should also be investigated. Cheaper controllers are prone to oscillation, therefore it is necessary to investigate how far a fluctuation hydroponic systems and aquaponic systems are willing to tolerate.

6. AI-based systems are difficult to develop due to the lack of availability of datasets. Therefore, there is a need for more datasets to allow researchers to develop efficient AI-based hydroponic systems.

\section{Conclusion}

In this paper smart hydroponic systems in the literature have been investigated. Table 1, 2, and 3 summarize the survey carried out in this paper. It is noted that researchers have done a lot of work in the monitoring of hydroponic systems and the automation of nutrient supply. However, the automation of nutrient supply is mainly controlled by proportional controllers, who are notorious for oscillations. The few AI-based research carried out have shown that AI is a good technique for controlling the hydroponic system, but they are expensive and require huge computation. Also, it is noted that IoT is the most attractive form of automation type, because of the flexibility it offers during system design. This is all the more reason why the security of the hydroponic system must be developed.

Conflict of Interest The authors declare no conflict of interest.

Acknowledgment The authors would like to thank the Tertiary Education Trust Fund (TETFund), Yobe State University (YSU) Damaturu Nigeria and King Fahd University of Petroleum and Minerals (KFUPM) for their support in this research. 


\section{References}

[1] T. Baras, DIY Hydroponic Gardens: How to Design and Build an Inexpensive System for Growing Plants in Water. Cool Springs Press, 2018. [Online]. Available: https://books.google.com.sa/books?id=rwlMDwAAQBAJ

[2] D. Singh, J. Davidson, and M. Books, Introduction to Hydroponics - Growing Your Plants Without Any Soil, ser. Gardening Series. Mendon Cottage Books, 2016. [Online]. Available: https://books.google.com.sa/books?id=RAMtDQAAQBAJ

[3] M. Raviv and J. Lieth, Soilless Culture: Theory and Practice. Elsevier Science, 2007. [Online]. Available: https://books.google.com.sa/books?id= NvDHJxRwsgYC

[4] W. Ke and Z. Xiong, "Difference of growth, copper accumulation and mineral element uptake in two elsholtzia haichowensis populations under copper and mineral nutrition stress," in 2008 2nd International Conference on Bioinformatics and Biomedical Engineering. IEEE, 2008, pp. 4704-4708.

[5] H. Wang, Y. Wang, and Y. Yang, "Effects of exogenous phenolic acids on roots of poplar hydroponic cuttings," in 2011 International Conference on Remote Sensing, Environment and Transportation Engineering. IEEE, 2011, pp. 8245-8249.

[6] N. Suzui, N. Kawachi, M. Yamaguchi, N. S. Ishioka, and S. Fujimaki, "A monitoring system of radioactive tracers in hydroponic solution for research on plant physiology," in 2009 1st International Conference on Advancements in Nuclear Instrumentation, Measurement Methods and their Applications. IEEE, 2009, pp. 1-3

[7] M. Liu, X. Xi, S. Wang, Y. Xu, and W. Song, "Research on differences of component and quantity of organic acids in the root exudates among the three green manures," in World Automation Congress 2012. IEEE, 2012, pp. 1-4.

[8] G. Gupta, Text Book of Plant Diseases. Discovery Publishing House, 2004. [Online]. Available: https://books.google.com.sa/books?id=OuoicDXQ-xYC

[9] T. Kaewwiset and T. Yooyativong, "Estimation of electrical conductivity and ph in hydroponic nutrient mixing system using linear regression algorithm," in 2017 International Conference on Digital Arts, Media and Technology (ICDAMT). IEEE, 2017, pp. 1-5.

[10] J. Muro, I. Irigoyen, P. Samitier, P. Mazuela, M. Salas, J. Soler, and M. Urrestarazu, "Wood fiber as growing medium in hydroponic crop," in International Symposium on Soilless Culture and Hydroponics 697, 2004, pp. 179-185.

[11] A.-K. Ammar, A. Anas, D. Ehab, and H. Yazan, "Hydroponics system," pp. 14-16, 2019. [Online]. Available: https://www.researchgate.net/publication/ 330578599_Hydroponics_Integrated_with_PV_System

[12] M. Honeydew, Gardening. Lulu.com, 2016. [Online]. Available: https://books.google.com.sa/books?id=4EMyDAAAQBAJ

[13] J. D. Butcher, C. P. Laubscher, and J. C. Coetzee, "A study of oxygenation techniques and the chlorophyll responses of pelargonium tomentosum grown in deep water culture hydroponics," HortScience, vol. 52, no. 7, pp. 952-957, 2017.

[14] J. Jones, Hydroponics: A Practical Guide for the Soilless Grower. CRC Press, 2016. [Online]. Available: https: //books.google.com.ng/books?id=y_bKBQAAQBAJ

[15] N. JOSHI, GREEN SPACES: CREATE YOUR OWN. Notion Press, 2018. [Online]. Available: https://books.google.com.sa/books?id=CA9tDwAAQBAJ

[16] G. Van Patten, Hydroponic Basics. Van Patten Publishing, 2004. [Online] Available: https://books.google.com.sa/books?id=L87bl7EIYkkC

[17] M. Calvin, Foundations of Space Biology and Medicine. Volume 3: Space Medicine and Biotechnology. National Aeronautics and Space Administration, 1975. [Online]. Available: https://books.google.com.sa/books?id=iOA_AQAAMAAJ

[18] M. C. Watson, “Fogponic plant growth system,” Dec. 27 2018, uS Patent App. 15/974,356.

[19] P. Wootton-Beard, "Growing without soil: An overview of hydroponics," Farming Connect, 2019. [Online]. Available: https://pure.aber.ac.uk/portal/ files/30769801/technical_article_hydroponics_final.pdf
[20] P. Sambo, C. Nicoletto, A. Giro, Y. Pii, F. Valentinuzzi, T. Mimmo, P. Lugli, G. Orzes, F. Mazzetto, S. Astolfi et al., "Hydroponic solutions for soilless production systems: Issues and opportunities in a smart agriculture perspective," Frontiers in plant science, vol. 10, 2019.

[21] O. Adrianes and G. M. S. Zarazúa, "Potassium acrylate: A novelty in hydroponic substrates," in 2017 XIII International Engineering Congress (CONIIN). IEEE, 2017, pp. 1-8.

[22] A. N. Harun, R. Ahmad, and N. Mohamed, "Plant growth optimization using variable intensity and far red led treatment in indoor farming," in 2015 International Conference on Smart Sensors and Application (ICSSA). IEEE, 2015, pp. 92-97.

[23] S. Huo, J. Liu, M. Addy, P. Chen, D. Necas, P. Cheng, K. Li, H. Chai, Y. Liu, and R. Ruan, "The influence of microalgae on vegetable production and nutrient removal in greenhouse hydroponics," Journal of Cleaner Production, vol. 243, p. 118563, 2020. [Online]. Available: http://www.sciencedirect.com/science/article/pii/S095965261933433X

[24] D. Savvas, "SW-soil and water: Automated replenishment of recycled greenhouse effluents with individual nutrients in hydroponics by means of two alternative models," Biosystems Engineering, vol. 83, no. 2, pp. 225 - 236, 2002. [Online]. Available: http://www.sciencedirect.com/science/article/pii/S1537511002901066

[25] D. S. Domingues, H. W. Takahashi, C. A. Camara, and S. L. Nixdorf, "Automated system developed to control ph and concentration of nutrient solution evaluated in hydroponic lettuce production," Computers and electronics in agriculture, vol. 84, pp. 53-61, 2012

[26] A. Phutthisathian, N. Pantasen, and N. Maneerat, "Ontology-based nutrient solution control system for hydroponics," in 2011 First International Conference on Instrumentation, Measurement, Computer, Communication and Control. IEEE, 2011, pp. 258-261.

[27] S. YAMAGUCHI, Y. TAKAHASHI, and T. HAYASHI, "Small indoor hydroponic system with renewable energy," in 2018 18th International Conference on Control, Automation and Systems (ICCAS). IEEE, 2018, pp. 313-318.

[28] M. F. Saaid, N. Fadhil, M. M. Ali, and M. Noor, "Automated indoor aquaponic cultivation technique," in 2013 IEEE 3rd International Conference on System Engineering and Technology. IEEE, 2013, pp. 285-289.

[29] D. T. C. Tu, P. B. Duong, N. T. H. Vuong, P. H. Luan, N. H. Nghia, P. N. Dien, N. H. Quang, and C. H. Buu, "The cactus device - an ultimate solution for irrigation," in 2016 3rd International Conference on Green Technology and Sustainable Development (GTSD), Nov 2016, pp. 270-273.

[30] E. Saenz, M. Jimenez, and A. Ramirez, "Strawberries collecting robot prototype in greenhouse hydroponic systems," in Symposium of Signals, Images and Artificial Vision-2013: STSIVA-2013. IEEE, 2013, pp. 1-4.

[31] Y. Takeuchi, "3d printable hydroponics: A digital fabrication pipeline for soilless plant cultivation,” IEEE Access, vol. 7, pp. 35 863-35 873, 2019.

[32] Y. Takeuchi, "Printable hydroponic gardens: Initial explorations and considerations," in Proceedings of the 2016 CHI Conference Extended Abstracts on Human Factors in Computing Systems, ser. CHI EA '16. New York, NY, USA: ACM, 2016, pp. 449-458. [Online]. Available: http://doi.acm.org/10.1145/2851581.2892587

[33] _ - "Printable hydroponics: Digital fabrication of ecological systems," in Proceedings of the 2018 ACM International Conference on Interactive Surfaces and Spaces, ser. ISS '18. New York, NY, USA: ACM, 2018, pp. 433-435. [Online]. Available: http://doi.acm.org/10.1145/3279778.3279929

[34] N. Seemuang, "A cleaning robot for greenhouse roofs," in 2017 2nd International Conference on Control and Robotics Engineering (ICCRE). IEEE, 2017, pp. 49-52.

[35] M. Sekimoto, K. Ikeshiro, and H. Imamura, "Development of interface using marker less ar for hydroponic culture managing systems in the distant place," in 2017 IEEE 12th International Conference on Power Electronics and Drive Systems (PEDS). IEEE, 2017, pp. 843-847.

[36] D. Eridani, O. Wardhani, and E. D. Widianto, "Designing and implementing the arduino-based nutrition feeding automation system of a prototype scaled nutrient film technique (nft) hydroponics using total dissolved solids (tds) sensor," in 2017 4th International Conference on Information Technology, Computer, and Electrical Engineering (ICITACEE). IEEE, 2017, pp. 170-175. 
[37] C. Peuchpanngarm, P. Srinitiworawong, W. Samerjai, and T. Sunetnanta, "Diy sensor-based automatic control mobile application for hydroponics," in 2016 Fifth ICT International Student Project Conference (ICT-ISPC). IEEE, 2016, pp. 57-60.

[38] N. K. Jacob, "Iot powered portable aquaponics system," in Proceedings of the Second International Conference on Internet of Things, Data and Cloud Computing, ser. ICC'17. New York, NY, USA: ACM, 2017, pp. 66:1-66:5. [Online]. Available: http://doi.acm.org/10.1145/3018896.3018965

[39] T. Changmai, S. Gertphol, and P. Chulak, "Smart hydroponic lettuce farm using internet of things," in 2018 10th International Conference on Knowledge and Smart Technology (KST). IEEE, 2018, pp. 231-236.

[40] G. Dbritto and S. Hamdare, "An ai based system design to develop and monitor a hydroponic farm," in 2018 International Conference on Smart City and Emerging Technology (ICSCET), Jan 2018, pp. 1-5.

[41] A. Nursyahid, M. R. Wibisono, E. D. Wardihani, T. A. Setyawan et al., "Plant age identification system of outdoor hydroponic cultivation based on digital image processing," in 2017 4th International Conference on Information Technology, Computer, and Electrical Engineering (ICITACEE). IEEE, 2017, pp. 213-218.

[42] K. Wongpatikaseree, N. Hnoohom, and S. Yuenyong, "Machine learning methods for assessing freshness in hydroponic produce," in 2018 International Joint Symposium on Artificial Intelligence and Natural Language Processing (iSAI-NLP). IEEE, 2018, pp. 1-4.

[43] T. Namgyel, S. Siyang, C. Khunarak, T. Pobkrut, J. Norbu, T. Chaiyasit, and T. Kerdcharoen, "Iot based hydroponic system with supplementary led light for smart home farming of lettuce," in 2018 15th International Conference on Electrical Engineering/Electronics, Computer, Telecommunications and Information Technology (ECTI-CON). IEEE, 2018, pp. 221-224.

[44] S. Tagle, R. Pena, F. Oblea, H. Benoza, N. Ledesma, J. Gonzaga, and L. A. G. Lim, "Development of an automated data acquisition system for hydroponic farming," in 2018 IEEE 10th International Conference on Humanoid, Nanotechnology, Information Technology, Communication and Control, Environment and Management (HNICEM), Nov 2018, pp. 1-5.

[45] P. Sihombing, N. A. Karina, J. T. Tarigan, and M. I. Syarif, "Automated hydroponics nutrition plants systems using arduino uno microcontroller based on android," Journal of Physics: Conference Series, vol. 978, p. 012014, mar 2018. [Online]. Available: https://doi.org/10.1088\%2F1742-6596\%2F978\%2F1\%2F012014

[46] P. Belhekar, A. Thakare, P. Budhe, U. Shinde, and V. Waghmode, "Automated system for farming with hydroponic style," in 2018 Fourth International Conference on Computing Communication Control and Automation (ICCUBEA), Aug 2018, pp. 1-4.

[47] F. Jaimes, B. Collazos, E. Arce, and M. Chauca, "Hydroponic system with automated hydrolysis using renewable energy self-sustainable," in MATEC Web of Conferences, vol. 256. EDP Sciences, 2019, p. 02012.

[48] C. Cambra, S. Sendra, J. Lloret, and R. Lacuesta, "Smart system for bicarbonate control in irrigation for hydroponic precision farming," Sensors, vol. 18, no. 5, p. 1333, 2018.

[49] R. E. N. Sisyanto, N. B. Kurniawan et al., "Hydroponic smart farming using cyber physical social system with telegram messenger," in 2017 International Conference on Information Technology Systems and Innovation (ICITSI). IEEE, 2017, pp. 239-245.

[50] M. Saaid, N. Yahya, M. Noor, and M. M. Ali, "A development of an automatic microcontroller system for deep water culture (dwc)," in 2013 IEEE 9th International Colloquium on Signal Processing and its Applications. IEEE, 2013, pp. 328-332.

[51] M. F. Saaid, A. Sanuddin, M. Ali, and M. S. A. I. M. Yassin, "Automated ph controller system for hydroponic cultivation," in 2015 IEEE Symposium on Computer Applications Industrial Electronics (ISCAIE), April 2015, pp. 186-190.

[52] W. Martindale and P. S. of Great Britain, The extra pharmocopoeia, ser The extra pharmocopoeia. Pharmaceutical Press, 1955, no. v. 2. [Online] Available: https://books.google.com.sa/books?id=8cxQAAAAYAAJ

[53] T. Nishimura, Y. Okuyama, and A. Satoh, "High-accuracy and low-cost sensor module for hydroponic culture system," in 2016 IEEE 5th Global Conference on Consumer Electronics. IEEE, 2016, pp. 1-4.
[54] T. Nishimura, Y. Okuyama, A. Matsushita, H. Ikeda, and A. Satoh, "A compact hardware design of a sensor module for hydroponics," in 2017 IEEE 6th Global Conference on Consumer Electronics (GCCE). IEEE, 2017, pp. 1-4.

[55] L. Velazquez, M. Hernandez, M. Leon, R. B. Domínguez, and J. Gutierrez, "First advances on the development of a hydroponic system for cherry tomato culture," in 2013 10th International Conference on Electrical Engineering, Computing Science and Automatic Control (CCE). IEEE, 2013, pp. 155-159.

[56] S. Charumathi, R. Kaviya, J. Kumariyarasi, R. Manisha, and P. Dhivya, "Optimization and control of hydroponics agriculture using iot," Asian J. Appl. Sci. Technol, vol. 1, no. 2, pp. 96-98, 2017.

[57] R. Nalwade and T. Mote, "Hydroponics farming," in 2017 International Conference on Trends in Electronics and Informatics (ICEI). IEEE, 2017, pp. $645-650$.

[58] A. M. Nagayo, C. Mendoza, E. Vega, R. K. Al Izki, and R. S. Jamisola, "An automated solar-powered aquaponics system towards agricultural sustainability in the sultanate of oman," in 2017 IEEE International Conference on Smart Grid and Smart Cities (ICSGSC). IEEE, 2017, pp. 42-49.

[59] F. Aliyu, S. umar, and H. Al-Duwaish, "A survey of applications of artificial neural networks in wireless sensor networks," 2019, the 8th International Conference on Modeling, Simulation and Applied Optimization (ICMSAO'2019).

[60] F. Aliyu and T. Sheltami, "Development of an energy-harvesting toxic and combustible gas sensor for oil and gas industries," Sensors and Actuators B: Chemical, vol. 231, pp. 265 - 275, 2016. [Online]. Available: http://www.sciencedirect.com/science/article/pii/S0925400516303318

[61] F. Aliyu, M. Al-shaboti, Y. Garba, T. Sheltami, A. Barnawi, and M. A. Morsy, "Hydrogen sulfide (h2s) gas safety system for oil drilling sites using wireless sensor network," Procedia Computer Science, vol. 63, pp. 499 - 504 2015, the 6th International Conference on Emerging Ubiquitous Systems and Pervasive Networks (EUSPN 2015)/ The 5th International Conference on Current and Future Trends of Information and Communication Technologies in Healthcare (ICTH-2015)/ Affiliated Workshops. [Online]. Available: http://www.sciencedirect.com/science/article/pii/S1877050915025107

[62] J. Ko, C. Lu, M. B. Srivastava, J. A. Stankovic, A. Terzis, and M. Welsh, "Wireless sensor networks for healthcare," Proceedings of the IEEE, vol. 98 , no. 11, pp. 1947-1960, 2010.

[63] M. A. Hussain, K. kyung Sup et al., "Wsn research activities for military application," in 2009 11th International Conference on Advanced Communication Technology, vol. 1. IEEE, 2009, pp. 271-274.

[64] Y. Wang, X. Yin, and D. You, "Application of wireless sensor networks in smart grid," power System technology, vol. 34, no. 5, pp. 7-11, 2010.

[65] R. Hussain, J. Sahgal, P. Mishra, and B. Sharma, "Application of wsn in rural development, agriculture water management," International Journal of Soft Computing and Engineering (IJSCE), vol. 2, no. 5, pp. 68-72, 2012.

[66] N. Jai, B. Dontha, A. Tripathy, and S. Mande, "Near real time-sensing system for hydroponics based urban farming," in 2018 3rd International Conference for Convergence in Technology (I2CT). IEEE, 2018, pp. 1-5.

[67] K. Kalovrektis, C. Lykas, I. Fountas, A. Gkotsinas, I. Lekakis et al., "Development and application embedded systems and wireless network of sensors to control of hydroponic greenhouses," International Journal of Agriculture and Forestry, vol. 3, no. 5, pp. 198-202, 2013.

[68] R. Vidhya and K. Valarmathi, "Survey on automatic monitoring of hydroponics farms using iot," in 2018 3rd International Conference on Communication and Electronics Systems (ICCES). IEEE, 2018, pp. 125-128.

[69] M. B. Fernandes, B. A. Costa, and J. M. Lemos, "Hydroponic greenhouse crop optimization," in 2018 13th APCA International Conference on Control and Soft Computing (CONTROLO). IEEE, 2018, pp. 270-275.

[70] B. Siregar, S. Efendi, H. Pranoto, R. Ginting, U. Andayani, and F. Fahmi, "Remote monitoring system for hydroponic planting media," in 2017 International Conference on ICT For Smart Society (ICISS). IEEE, 2017, pp. $1-6$.

[71] K. S. Aishwarya, M. Harish, S. Prathibhashree, and K. Panimozhi, "Survey on iot based automated aquaponics gardening approaches," in 2018 Second International Conference on Inventive Communication and Computational Technologies (ICICCT), April 2018, pp. 1495-1500. 
[72] T.-H. Wu, C.-H. Chang, Y.-W. Lin, L.-D. Van, and Y.-B. Lin, "Intelligent plant care hydroponic box using iottalk," in 2016 IEEE International Conference on Internet of Things (iThings) and IEEE Green Computing and Communications (GreenCom) and IEEE Cyber, Physical and Social Computing (CPSCom) and IEEE Smart Data (SmartData). IEEE, 2016, pp. 398-401.

[73] Y.-B. Lin, Y.-W. Lin, C.-M. Huang, C.-Y. Chih, and P. Lin, "Iottalk: A management platform for reconfigurable sensor devices," IEEE Internet of Things Journal, vol. 4, no. 5, pp. 1552-1562, 2017.

[74] W.-L. Chen, Y.-B. Lin, Y.-W. Lin, R. Chen, J.-K. Liao, F.-L. Ng, Y.-Y. Chan, Y.-C. Liu, C.-C. Wang, C.-H. Chiu et al., "Agritalk: Iot for precision soil farming of turmeric cultivation," IEEE Internet of Things Journal, 2019.

[75] L.-D. Van, Y.-B. Lin, T.-H. Wu, Y.-W. Lin, S.-R. Peng, L.-H. Kao, and C.-H. Chang, "Planttalk: A smartphone-based intelligent hydroponic plant box," Sensors, vol. 19, no. 8, p. 1763, 2019.

[76] M. A. G. Maureira, D. Oldenhof, and L. Teernstra, "Thingspeak-an api and web service for the internet of things," World Wide Web, 2011.

[77] K. E. Lakshmiprabha and C. Govindaraju, "Hydroponic-based smart irrigation system using internet of things," International Journal of Communication Systems, p. e4071, 2019.

[78] P. N. Crisnapati, I. N. K. Wardana, I. K. A. A. Aryanto, and A. Hermawan, "Hommons: Hydroponic management and monitoring system for an iot based nft farm using web technology," in 2017 5th International Conference on Cyber and IT Service Management (CITSM), Aug 2017, pp. 1-6.

[79] P. N. Crisnapati, I. N. K. Wardana, I. K. A. A. Aryanto, and A. Hermawan, "Hommons: Hydroponic management and monitoring system for an iot based nft farm using web technology," in 2017 5th International Conference on Cyber and IT Service Management (CITSM). IEEE, 2017, pp. 1-6.

[80] G. J. U. Ossa, C. G. Montoya, and M. A. V. Velásquez, "Control and monitoring of a stevia plantation in a hydroponic system," in 2017 IEEE $3 \mathrm{rd}$ Colombian Conference on Automatic Control (CCAC), Oct 2017, pp. 1-5.

[81] M. A. Triawan, H. Hindersah, D. Yolanda, and F. Hadiatna, "Internet of things using publish and subscribe method cloud-based application to nftbased hydroponic system," in 2016 6th International Conference on System Engineering and Technology (ICSET). IEEE, 2016, pp. 98-104.

[82] S. Ruengittinun, S. Phongsamsuan, and P. Sureeratanakorn, "Applied internet of thing for smart hydroponic farming ecosystem (hfe)," in 2017 10th International Conference on Ubi-media Computing and Workshops (Ubi-Media), Aug 2017, pp. 1-4.

[83] A. Satoh, "A hydroponic planter system to enable an urban agriculture service industry," in 2018 IEEE 7th Global Conference on Consumer Electronics (GCCE). IEEE, 2018, pp. 281-284

[84] J. Chaiwongsai, "Automatic control and management system for tropical hydroponic cultivation," in 2019 IEEE International Symposium on Circuits and Systems (ISCAS), May 2019, pp. 1-4.

[85] F. Aliyu, T. Sheltami, and E. M. Shakshuki, "A detection and prevention technique for man in the middle attack in fog computing," Procedia Computer Science, vol. 141, pp. 24-31, 2018.

[86] M. A. Zamora-Izquierdo, J. Santa, J. A. Martínez, V. Martínez, and A. F. Skarmeta, "Smart farming iot platform based on edge and cloud computing," Biosystems Engineering, vol. 177, pp. 4 - 17, 2019, intelligent Systems for Environmental Applications. [Online]. Available: http://www.sciencedirect.com/science/article/pii/S1537511018301211

[87] I. Batyrshin, M. de Lourdes Martínez-Villaseñor, and H. Espinosa, Advances in Computational Intelligence: 17th Mexican International Conference on Artificial Intelligence, MICAI 2018, Guadalajara, Mexico, October 22-27, 2018, Proceedings, ser. Lecture Notes in Computer Science. Springer International Publishing, 2019, no. pt. 2. [Online]. Available: https://books.google.com.ng/books?id=PUOCDwAAQBAJ

[88] I. Boiko, Non-parametric Tuning of PID Controllers: A Modified Relay-Feedback-Test Approach, ser. Advances in Industrial Control. Springer London, 2012. [Online]. Available: https://books.google.com.sa/books?id=vE6PHWgnRgsC
[89] M. Chidambaram and N. Saxena, Relay Tuning of PID Controllers: For Unstable MIMO Processes, ser. Advances in Industrial Control. Springer Singapore, 2018. [Online]. Available: https://books.google.com.sa/books?id=GGRLDwAAQBAJ

[90] J. A. Asumadu, B. Smith, N. S. Dogan, P. A. Loretan, and H. Aglan, "Microprocessor-based instrument for hydroponic growth chambers used in ecological life support systems," in Quality Measurement: The Indispensable Bridge between Theory and Reality (No Measurements? No Science! Joint Conference-1996: IEEE Instrumentation and Measurement Technology Conference and IMEKO Tec, vol. 1. IEEE, 1996, pp. 325-329.

[91] M. F. Saaid, A. Sanuddin, M. Ali, and M. S. A. I. M. Yassin, "Automated $\mathrm{pH}$ controller system for hydroponic cultivation," in 2015 IEEE Symposium on Computer Applications Industrial Electronics (ISCAIE), April 2015, pp. 186-190.

[92] S. Adhau, R. Surwase, and K. H. Kowdiki, "Design of fully automated low cost hydroponic system using labview and avr microcontroller," in 2017 IEEE International Conference on Intelligent Techniques in Control, Optimization and Signal Processing (INCOS), March 2017, pp. 1-4.

[93] S. Gertphol, P. Chulaka, and T. Changmai, "Predictive models for lettuce quality from internet of things-based hydroponic farm," in 2018 22nd International Computer Science and Engineering Conference (ICSEC). IEEE, 2018, pp. 1-5.

[94] M. I. Alipio, A. E. M. D. Cruz, J. D. A. Doria, and R. M. S. Fruto, "On the design of nutrient film technique hydroponics farm for smart agriculture," Engineering in Agriculture, Environment and Food, vol. 12, no. 3, pp. 315 - 324, 2019. [Online]. Available: http://www.sciencedirect.com/science/article/pii/S1881836617303294

[95] S. Mashumah, M. Rivai, and A. N. Irfansyah, "Nutrient film technique based hydroponic system using fuzzy logic control," in 2018 International Seminar on Intelligent Technology and Its Applications (ISITIA). IEEE, 2018, pp. 387-390.

[96] D. Yolanda, L. Arief, T. A. Sundara, M. Deza, and D. Oktavia, "Control of electrical conductivity for nft hydroponic systems using fuzzy logic and android environment," in 2018 International Conference on Information Technology Systems and Innovation (ICITSI). IEEE, 2018, pp. 508-514.

[97] T. A. Setyawan, S. A. Riwinanto, Suhendro, Helmy, A. Nursyahid, and A. S. Nugroho, "Comparison of hsv and lab color spaces for hydroponic monitoring system," in 2018 5th International Conference on Information Technology, Computer, and Electrical Engineering (ICITACEE), Sep. 2018, pp. 347-352.

[98] D. Yolanda, H. Hindersah, F. Hadiatna, and M. A. Triawan, "Implementation of real-time fuzzy logic control for nft-based hydroponic system on internet of things environment," in 2016 6th International Conference on System Engineering and Technology (ICSET). IEEE, 2016, pp. 153-159.

[99] L. M. JSM and C. Sridevi, "Design of efficient hydroponic nutrient solution control system using soft computing based solution grading," in 2014 International Conference on Computation of Power, Energy, Information and Communication (ICCPEIC). IEEE, 2014, pp. 148-154.

[100] T. Ayala-Silva and C. A. Beyl, "Use of neural networks to discriminate between control leaves of wheat or those deficient in nitrogen, phosphorus, potassium, and calcium using spectral data," in Proceedings of the 5th Biannual World Automation Congress, vol. 13, June 2002, pp. 49-57.

[101] T. Morimoto, I. Cho, and Y. Hashimoto, "Identification of hydroponics in an advanced control system of the greenhouse," IFAC Proceedings Volumes, vol. 25, no. 15, pp. 169 - 174, 1992, 9th IFAC/IFORS Symposium on Identification and System Parameter Estimation 1991 , Budapest, Hungary, 8-12 July 1991. [Online]. Available: http://www.sciencedirect.com/science/article/pii/S147466701750628X

[102] T. Morimoto, T. Takeuchi, and Y. Hashimoto, "Growth optimization of plant by means of the hybrid system of genetic algorithm and neural network," in Proceedings of 1993 International Conference on Neural Networks (IJCNN93-Nagoya, Japan), vol. 3. IEEE, 1993, pp. 2979-2982. 